\title{
Studies on Knot Placement Techniques for the Geometry Construction and the Accurate Simulation of Isogeometric Spatial Curved Beams
}

\author{
Seyed Farhad Hosseini ${ }^{1}$, Ali Hashemian ${ }^{2,3 *}$, Alessandro Reali ${ }^{4}$ \\ 1. Sun-Air Research Institute, Ferdowsi University of Mashhad, Mashhad, Iran \\ 2. BCAM - Basque Center for Applied Mathematics, Bilbao, Basque Country, Spain \\ 3. Department of Mechanical Engineering, Hakim Sabzevari University, Sabzevar, Iran \\ 4. Department of Civil Engineering and Architecture, University of Pavia, Pavia, Italy \\ * Corresponding author, email address: a.hashemian@hsu.ac.ir, Tel/Fax.: +98 5144012819
}

\begin{abstract}
The present paper investigates the use of different knot placement techniques for isogeometric analysis of spatial curved beams, to enhance analysis results in cases when geometries are given in terms of data points. Focusing on analysis-aware modeling for structural static and vibration simulations of spatial free-form curved beams, the knot placement techniques based on uniformly spaced knots as well as on De Boor's and Piegl and Tiller's algorithms are studied. For this purpose, an isogeometric formulation for linear EulerBernoulli beams based on the Euler-Rodriguez transformation rule is implemented. Different case studies and numerical examples are presented and the results are validated against "overkill" solutions computed with a commercial finite element software. The results show that the De Boor's knot placement algorithm typically leads to better approximation errors and is therefore the suggested strategy for this kind of problems.
\end{abstract}

Keywords: Isogeometric analysis; analysis-aware modeling; curve approximation; knot placement techniques; spatial free-form curved beams

\section{Introduction}

Computer-aided design (CAD) and finite element analysis (FEA) are two important aspects of the mechanical design of solids and structures. CAD geometries developed by designers and architects need to be converted to appropriate models for numerical analyses. However, constructing analysis-suitable models for FEA is the most time-consuming procedure that generally requires several remodeling steps. The concept of isogeometric analysis (IGA), first introduced by Hughes et al. [1], tries to bridge the gap between CAD and FEA by employing spline basis functions (already used to create CAD geometries) as finite element shape functions. Thanks to the accurate and efficient geometry representation of this framework,

Postprint accepted by Computer Methods in Applied Mechanics and Engineering, published online on doi.org/10.1016/j.cma.2019.112705 
IGA has been successfully implemented in various engineering applications such as solid mechanics [2-6], fluid mechanics [7-10], heat transfer [11, 12] and eigenvalue problems [13, 14].

Isogeometric static and vibration analyses of curved beam structures have been taken into consideration by many researchers in recent years. We can categorize these research works into the two main groups of planar (see, e.g., [15-21]) and spatial (see, e.g., [22-27]) curved beam representations where the latter is more applicable in real-world engineering problems but needs a more complex formulation. Reviewing some recent works in this area, Bauer et al. [23] have proposed a continuum element formulation for static analysis of geometrically nonlinear space curved beams assuming Euler-Bernoulli theory. They have adopted the Euler-Rodriguez transformation law between undeformed and deformed beam configurations. Zhang et al. [24], considering locking issues, have proposed a linear element formulation for spatial beams based on the Frenet-Serret orientation frame. Radenković and Borković [25] have presented a linear elastic IGA for large displacement and small strain theories considering a new coordinate line that is orthogonal to the normal plane of the beam axis at each point. Isogeometric collocation of geometrically exact shear deformable beams and Cosserat rods are also studied by Marino [28] and Weeger et al. [29], respectively.

The present paper contributes to the field of IGA of space free-form curved beams. For this purpose, we implement a linear IGA formulation for Euler-Bernoulli beams based on Euler-Rodriguez transformation rule [23]. The main focus of this research is however on constructing suitable isogeometric parameterizations which can be categorized in the field of "analysis-aware modeling" (proposed by Cohen et al. [30] and extensively used in IGA by, e.g., Xu et al. [31-33], Casquero et al. [34] and Aigner et al. [35]). It is important to notice that the beam geometry for isogeometric analysis can be obtained either by direct input from commercial CAD software (like, e.g., Rhino) or by fitting a curve to a set of data points (which is the typical case in a number of applications [36]). In the case of direct geometry input, all necessary information such as the coordinates of control points and the parameterization of the curve are imported from the CAD system. For this type of problems, Hosseini et al. [37] recently employed a curve reparameterization technique to resolve the probable ill-conditioning issue of nonlinearly parameterized input B-spline geometries. On the other hand, when the beam geometry is given only in terms of input data points, in order to construct the geometry for IGA, the unknown control points need to be found by a curve fitting approach through interpolation or approximation through, e.g., a least-squares minimization. Two main steps that must be considered before fitting a curve to the input dataset are parameter selection and knot vector generation [38], that may significantly affect the constructed geometry and, therefore, the IGA solution outputs (e.g., the beam deflection and natural frequencies). The effect of different parameterization schemes on IGA results of planar curved beams has been investigated in detail by Hosseini et al. [39] where the chord-length parameter selection method - that results in an almost linear parameterization - is shown to be the most accurate approach. Cottrell et al. [40] presented the control point placement method to improve the IGA results of straight structures. The importance of one-to-one correspondence between parameterizations of the geometry, loading and rigidity of planar free-form curved beams are also addressed in [41]. In the aforementioned researches, it is shown that linear parameterization can lead to accurate IGA results. However, until very recently, the effect of different knot vector generation strategies on the IGA 
results has not been discussed in the literature. Employing different knot vector generation or so-called "knot placement" techniques not only leads to different least-squares errors in curve approximation, but also affects the mesh quality and the accuracy of IGA results. Considering the control point placement approach described in [40], it should be noted that, in the case of spatial free-form curved beams, the control points are obtained by a curve fitting process and any manipulation of their positions (like what is presented in [40]) will change the shape of the beam geometry which is not of interest for the context herein considered. It is also to be noted that an integrated approximation and knot placement approach with the aim of improving the fitting quality may need a relatively expensive optimization procedure with both control points and knots as unknowns (see Hashemian and Hosseini [42]) which is beyond the scope of this work.

The objective of this paper is to thoroughly address the different knot placement techniques in geometry construction for the isogeometric framework when the geometry is in terms of input data points. In this regard, three well-known knot placement techniques are studied - namely, uniformly spaced knots, De Boor's algorithm, and Piegl and Tiller's algorithm -, and their effects on isogeometric vibration and structural analysis results of spatial free-form curved beams are investigated. Hence, the remainder of the paper is organized as follows. In Section 2, the definition of B-spline curves (including the considered curve approximation procedure) is presented. The different knot vector generation approaches are presented in this section as well. The isogeometric formulation of spatial curved beams is introduced in Section 3. In Section 4, different case studies and numerical examples are presented along with comprehensive remarks on the accuracy of the results. Some supplementary investigations are performed in Section 5 to demonstrate how the knot placement techniques can deal with different geometric aspects of the IGA framework. Finally, Section 6 draws the conclusions.

\section{Geometry Construction by B-spline Curves}

B-splines can model a rich variety of free-form geometries and play a central role in the isogeometric analysis of free-form curved structures, where they are used both for representing the geometry and for expressing the displacement fields. They are also well consistent with commercial CAD software and employed in different engineering problems.

\subsection{B-spline Curve Definition}

A three-dimensional B-spline curve of degree $p$, is expressed as a piecewise continuous parametric function with $n+1$ control points $\boldsymbol{P}^{i}=\left[X^{i}, Y^{i}, Z^{i}\right]$ as follows [38]:

$$
\boldsymbol{r}(\xi)=\sum_{i=0}^{n} N_{i}^{p}(\xi) \boldsymbol{P}^{i} \quad(0 \leq \xi \leq 1)
$$

where $\boldsymbol{r}(\xi)=[x(\xi), y(\xi), z(\xi)]$ is a vector-valued function whose components are represented separately as mappings of the parameter $\xi \in[0,1]$ onto the $3 \mathrm{D}$ space. The univariate parameter space is characterized 
by the knot vector $\Xi$ as defined by Eq. (2). In addition, $N_{i}^{p}(\xi)$ is the $i$-th B-spline basis function of degree $p$ as expressed by Eq. (3) [38]:

$$
\begin{gathered}
\Xi=[\underbrace{0,0, \cdots, 0}_{p+1}, \xi_{p+1}, \xi_{p+2}, \cdots, \xi_{n}, \underbrace{1,1, \cdots, 1}_{p+1}] \\
N_{i}^{0}(\xi)=\left\{\begin{array}{cc}
1 & \xi_{i} \leq \xi<\xi_{i+1} \\
0 & \text { otherwise }
\end{array}\right. \\
N_{i}^{p}(\xi)=\frac{\xi-\xi_{i}}{\xi_{i+p}-\xi_{i}} N_{i}^{p-1}(\xi)+\frac{\xi_{i+p+1}-\xi}{\xi_{i+p+1}-\xi_{i+1}} N_{i+1}^{p-1}(\xi)
\end{gathered}
$$

For example, Fig. 1 depicts a 3D B-spline curve of degree three with eight control points and five uniformly spaced knot spans based on the knot vector $\Xi=[0,0,0,0,0.2,0.4,0.6,0.8,1,1,1,1]$, noting that for the case of non-repetitive and increasing internal knots (i.e., $\xi_{i}<\xi_{i+1}$ ), we have $n-p+1$ non-zero knot spans. This curve can be thought of as the geometry of a spatial curved beam. The $C^{2}$-continuous cubic basis functions and respective knots are shown in Fig. 2.

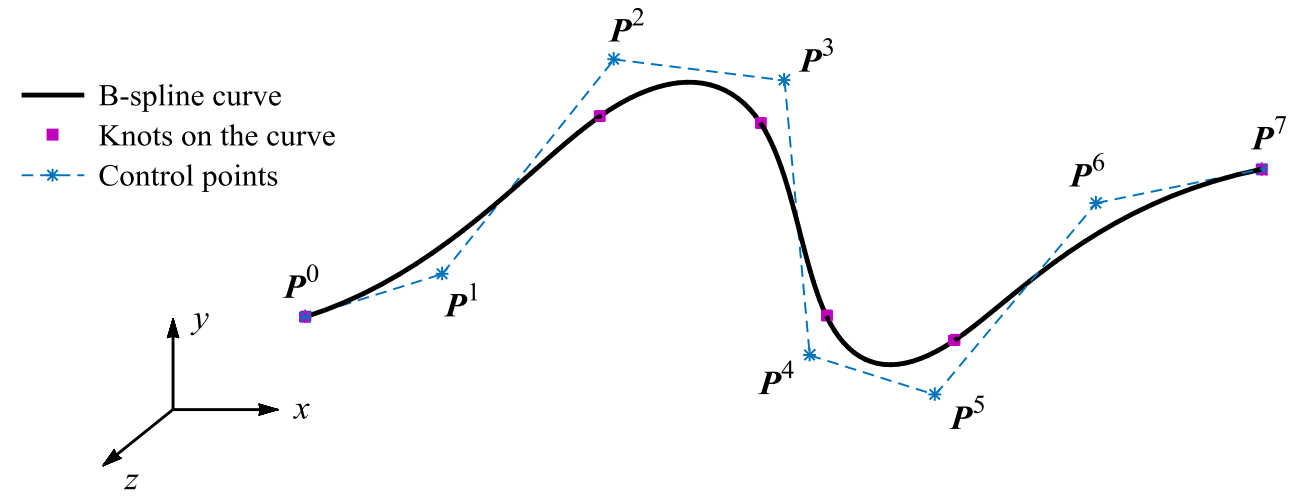

Fig. 1. A cubic B-spline curve in 3D space with eight control points and four internal knots

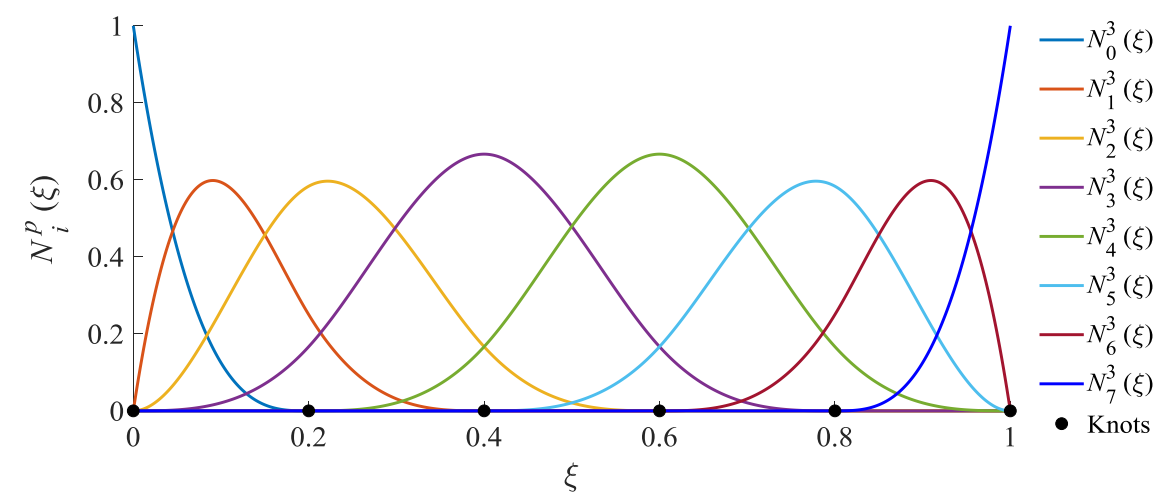

Fig. 2. Cubic basis functions and respective knots on the knot vector of B-spline curve of Fig. 1 


\subsection{B-spline Curve Fitting}

It is interesting to note that if only a set of data points representing the geometry of a space curve is available, the B-spline expression of the curve can be found by a curve fitting technique that is typically performed by curve interpolation, approximation or a combination of them [38]. There are some other optimizationbased approximations which rely on the conditions imposed on data points and/or on the overall curve profile (see, e.g., [42, 43]). As one of typical curve fitting approaches, interpolation guarantees the curve passes through all data points by setting the number of control points equal to data points. The fitted curve, however, may fluctuate rather than being smooth, especially for a noisy set of data points (see, e.g., [44]). The problem of fluctuating can be overcome in curve approximation by relaxing the strict requirement that all data points must be crossed by the curve. Except for the first and last points, the curve does not necessarily contain any other data point, but must track them with a minimum error. Finally, when the curve should exactly cross some data points, while other points can be tracked without the need of being exactly passed, a mixed interpolation-approximation procedure can be employed.

Focusing on curve approximation in this paper, given a set of $h+1$ data points $\boldsymbol{D}^{k}=\left[D_{x}^{k}, D_{y}^{k}, D_{z}^{k}\right]$ where $k=0,1, \cdots, h$, the curve is to be constructed in such a way that the control points are the output of a global curve fitting problem. In this regard, the first step is to associate the parameter $\bar{\xi}_{k}$ to the $k$-th data point $\boldsymbol{D}^{k}$ by imposing one of the uniformly spaced, chord-length, or centripetal parameter selection schemes described by Eqs. (4) to (6), respectively [38]:

$$
\begin{gathered}
\bar{\xi}_{k}=\frac{k}{h} \quad(k=0,1, \cdots, h) \\
\bar{\xi}_{0}=0 \quad, \quad \bar{\xi}_{k}=\frac{\sum_{i=1}^{k}\left\|\boldsymbol{D}^{i}-\boldsymbol{D}^{i-1}\right\|}{\sum_{i=1}^{h}\left\|\boldsymbol{D}^{i}-\boldsymbol{D}^{i-1}\right\|} \quad(k=1,2, \cdots, h) \\
\bar{\xi}_{0}=0 \quad, \quad \bar{\xi}_{k}=\frac{\sum_{i=1}^{k}\left\|\boldsymbol{D}^{i}-\boldsymbol{D}^{i-1}\right\|^{1 / 2}}{\sum_{i=1}^{h}\left\|\boldsymbol{D}^{i}-\boldsymbol{D}^{i-1}\right\|^{1 / 2}} \quad(k=1,2, \cdots, h)
\end{gathered}
$$

In the next step, an appropriate knot vector should be generated (see §2.3) and data points need to be approximated by a curve with $n+1$ control points $(n \leq h)$ where the first and last control points are simply determined as $\boldsymbol{P}^{0}=\boldsymbol{D}^{0}$ and $\boldsymbol{P}^{n}=\boldsymbol{D}^{h}$. Finally, the remaining control points $\boldsymbol{P}=\left[\boldsymbol{P}^{1}, \boldsymbol{P}^{2}, \cdots, \boldsymbol{P}^{n-1}\right]^{T}$ can be computed in the least-squares sense through the minimization of the fitting objective function (i.e., $L^{2}$ norm fitting error) $f=\sum_{k=1}^{h-1}\left\|\boldsymbol{D}^{k}-\boldsymbol{r}\left(\bar{\xi}_{k}\right)\right\|_{L^{2}}^{2}$. In this case, the number of control points is to be determined such that the desirable fitting error is achieved. By setting the derivatives $\partial f / \partial \boldsymbol{P}^{i}$ equal to zero, and employing standard matrix algebra, one obtains the control points as [38]:

$$
\boldsymbol{P}=\left(\boldsymbol{B}^{T} \boldsymbol{B}\right)^{-1} \boldsymbol{B}^{T} \boldsymbol{H}
$$

where 


$$
\boldsymbol{B}=\left[\begin{array}{cccc}
N_{1}^{p}\left(\bar{\xi}_{1}\right) & N_{2}^{p}\left(\bar{\xi}_{1}\right) & & N_{n-1}^{p}\left(\bar{\xi}_{1}\right) \\
N_{1}^{p}\left(\bar{\xi}_{2}\right) & N_{2}^{p}\left(\bar{\xi}_{2}\right) & \cdots & N_{n-1}^{p}\left(\bar{\xi}_{2}\right) \\
& \vdots & \ddots & \vdots \\
N_{1}^{p}\left(\bar{\xi}_{h-1}\right) & N_{2}^{p}\left(\bar{\xi}_{h-1}\right) & \cdots & N_{n-1}^{p}\left(\bar{\xi}_{h-1}\right)
\end{array}\right]
$$

and

$$
\boldsymbol{H}^{k}=\boldsymbol{D}^{k}-N_{0}^{p}\left(\bar{\xi}_{k}\right) \boldsymbol{D}^{0}-N_{n}^{p}\left(\bar{\xi}_{k}\right) \boldsymbol{D}^{h} \quad(k=1,2, \cdots, h-1)
$$

\subsection{Knot Placement Techniques for Geometry Construction}

In order for the geometry to be appropriately approximated by B-spline curves, the position of the internal knots of the knot vector is of central importance. Moreover, considering that elements in the isogeometric framework are corresponding to non-zero knot spans on the curve, the constructed geometry will affect the solution output as well. There are different knot vector generation techniques for curve/surface approximation in the literature (see, e.g., [42, 45-47]) where some well-known algorithms are revisited here. It should be pointed out that all mentioned knot placement techniques result in non-repetitive increasing internal knots on the knot vector (i.e., $\xi_{i}<\xi_{i+1}$ ). However, if multiple knots are of interest to lower the continuity at specific locations, the designer could add appropriate knots in a post-processing step. Geometries with sharp corners, which need $C^{0}$ continuity (i.e., multiplicity of order $p$ ) at the corner, may require additional considerations as discussed in [21,39].

\subsubsection{Uniform Knot Placement}

In the uniform knot placement technique, the internal knots are equally spaced in the $[0,1]$ interval. This is the easiest knot placement technique in curve approximation, which may result in some difficulties such as numerical instability of the geometry construction. In this method, referring to Eq. (2), the arrangement of the internal knots is simply [38]:

$$
\xi_{p+j}=\frac{j}{n-p+1} \quad(j=1,2, \cdots, n-p)
$$

\subsubsection{Knot Placement by De Boor's Algorithm}

The De Boor's algorithm is one of the most frequent knot placement techniques in curve reconstruction from input data that can bring in a stable and appropriate curve fitting. This method entails that, to guarantee every knot span contains at least one parameter $\bar{\xi}_{k}$, the internal knots should be defined as follows [38]:

$$
\xi_{p+j}=(1-\alpha) \bar{\xi}_{i-1}+\alpha \bar{\xi}_{i} \quad(j=1,2, \cdots, n-p)
$$

where by defining $\operatorname{int}($.$) as the floor function, we have:$ 


$$
\begin{gathered}
\alpha=j d-1 \\
i=\operatorname{int}(j d) \\
d=\frac{h+1}{n-p+1}
\end{gathered}
$$

\subsubsection{Knot Placement by Piegl and Tiller's Algorithm}

The third knot placement technique reviewed in this article is the algorithm presented by Piegl and Tiller [47]. This algorithm first groups $(h+1) /(n+1)$ consecutive parameters and averages them to find $n+1$

representative values $w_{i}(i=0,1, \cdots, n)$. Then, $p$ consecutive values are averaged to yield the knots. Based on this algorithm, the internal knots can be determined as:

$$
\xi_{p+j}=\frac{1}{p} \sum_{i=j}^{j+p-1} w_{i} \quad(j=1,2, \cdots, n-p)
$$

where

$$
w_{i}=\frac{1}{\alpha_{i}-\beta_{i}+1} \sum_{k=\beta_{i}}^{\alpha_{i}} \bar{\xi}_{k} \quad(i=0,1, \cdots, n)
$$

and

$$
\begin{aligned}
& \alpha_{i}=\operatorname{int}\left(d_{i}+0.5\right) \\
& \beta_{i}=\alpha_{i-1}+1 \quad\left(\beta_{0}=0\right) \\
& d_{i}=\frac{(i+1)(h+1)}{n+1}-1
\end{aligned}
$$

Fig. 3 illustrates the effect of different knot placement techniques on fitting a cubic curve to a planar dataset noting that the quality of the fitted curve would increase by employing more control points. The leastsquares fitting errors are quantified as 2.734, 1.687, and 2.195 for uniformly spaced knots, De Boor's and Piegl and Tiller's algorithms, respectively. The figure shows that in addition to the quality of the fitting process, the positions of control points and the values of their respective basis functions, which are important in IGA, also depend on the knot placement technique adopted for the geometry construction. It should be also pointed out that a good curve fit does not guarantee an accurate IGA solution as it will be discussed later in Section 4. 


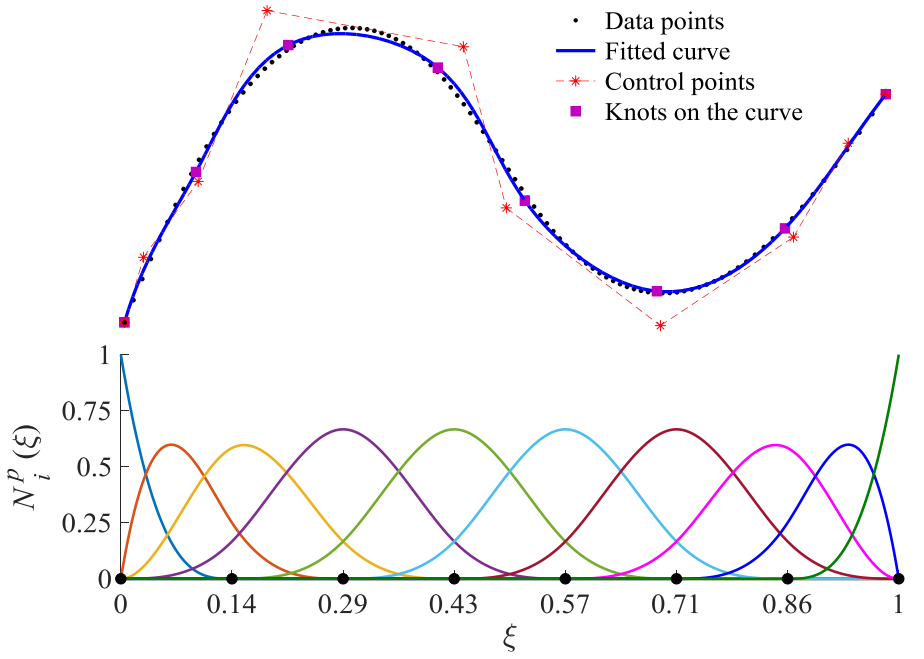

(a)
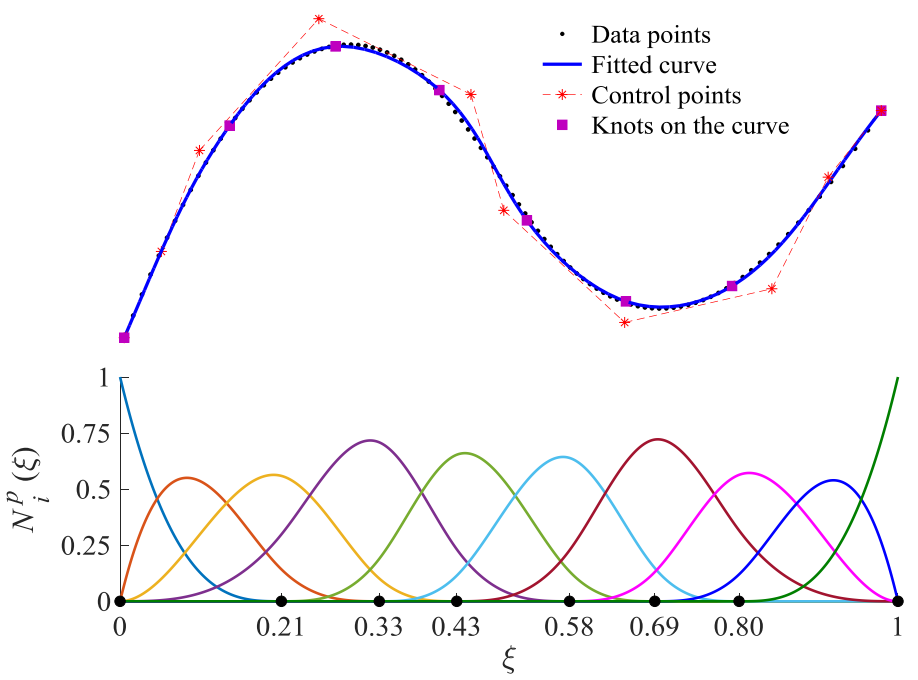

(b)
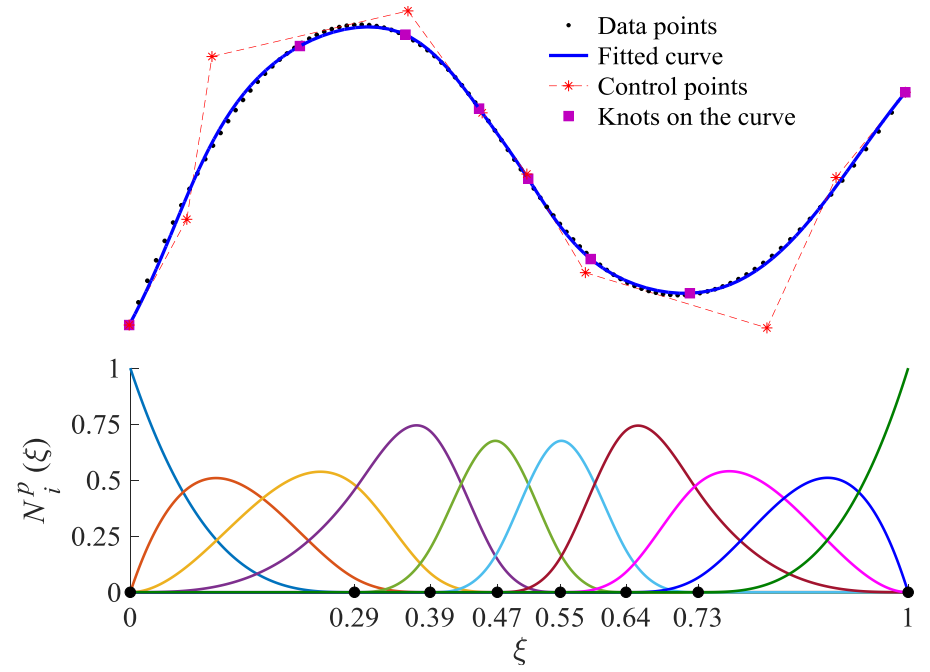

(c)

Fig. 3. Effect of different knot placement algorithms on curve fitting results and corresponding basis functions: (a) uniform, (b) De Boor, (c) Piegl and Tiller 


\section{Isogeometric Analysis of Spatial Curved Beams}

In this section, we present the adopted spatial curved beam formulation.

\subsection{Orientation Components in Space}

Given the parametric function $\boldsymbol{r}(\xi)=[x(\xi), y(\xi), z(\xi)]$ representing the geometry of a curved beam in space, in order to describe the kinematics of deformation, the orientation components of the geometry should be specified. The most frequent orthonormal frame associated with the orientation of a space curve is the Frenet frame (Fig. 4) defined by three pairwise orthogonal unit vectors as follows [48]:

$$
\begin{gathered}
\mathcal{T}(\xi)=\frac{\boldsymbol{r}^{\prime}(\xi)}{\left\|\boldsymbol{r}^{\prime}(\xi)\right\|} \\
\mathcal{B}(\xi)=\frac{\boldsymbol{r}^{\prime}(\xi) \times \boldsymbol{r}^{\prime \prime}(\xi)}{\left\|\boldsymbol{r}^{\prime}(\xi) \times \boldsymbol{r}^{\prime \prime}(\xi)\right\|} \\
\mathcal{N}(\xi)=\mathcal{B}(\xi) \times \mathcal{T}(\xi)
\end{gathered}
$$

$\boldsymbol{T}(\xi), \mathcal{N}(\xi)$ and $\mathcal{B}(\xi)$ are the unit tangent, normal and binormal vectors, respectively. Note that the binormal vector is perpendicular to the osculating plane of the curve spanned by the tangent and normal vectors.

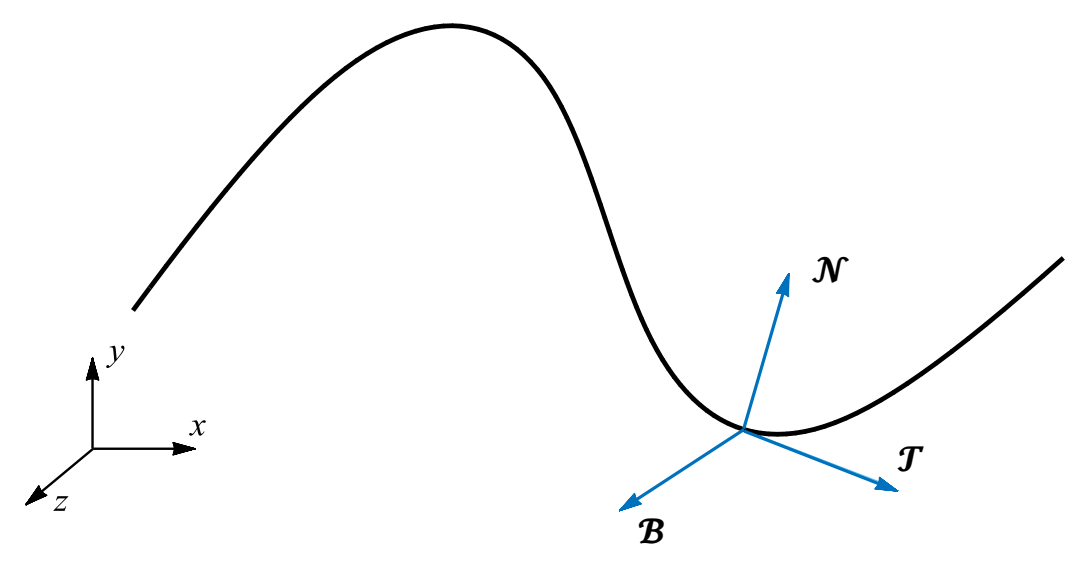

Fig. 4. Orthonormal Frenet frame on a spatial curve

In isogeometric formulation, it is necessary to compute the derivatives of the above-mentioned vectors which can be easily achieved by differentiating $\mathcal{T}(\xi), \mathcal{N}(\xi)$ and $\mathcal{B}(\xi)$ with respect to the curve parameter.

\subsection{Kinematics of Deformation}

In the current research, the convective curvilinear coordinate system, as described in Fig. 5, is employed for expressing the kinematics of spatial curved beams. The element formulation is based on Euler-Bernoulli beam theory assuming that the cross-section remains orthogonal to the centerline after deformation. It must 
be also noticed that the cross-section can undergo in-plane torsional shear deformation in spatial curved beams. Therefore, the deformed state of the beam can be characterized by four degrees of freedom (DOFs), which are three spatial displacement components and the in-plane cross-section rotation around the centerline.

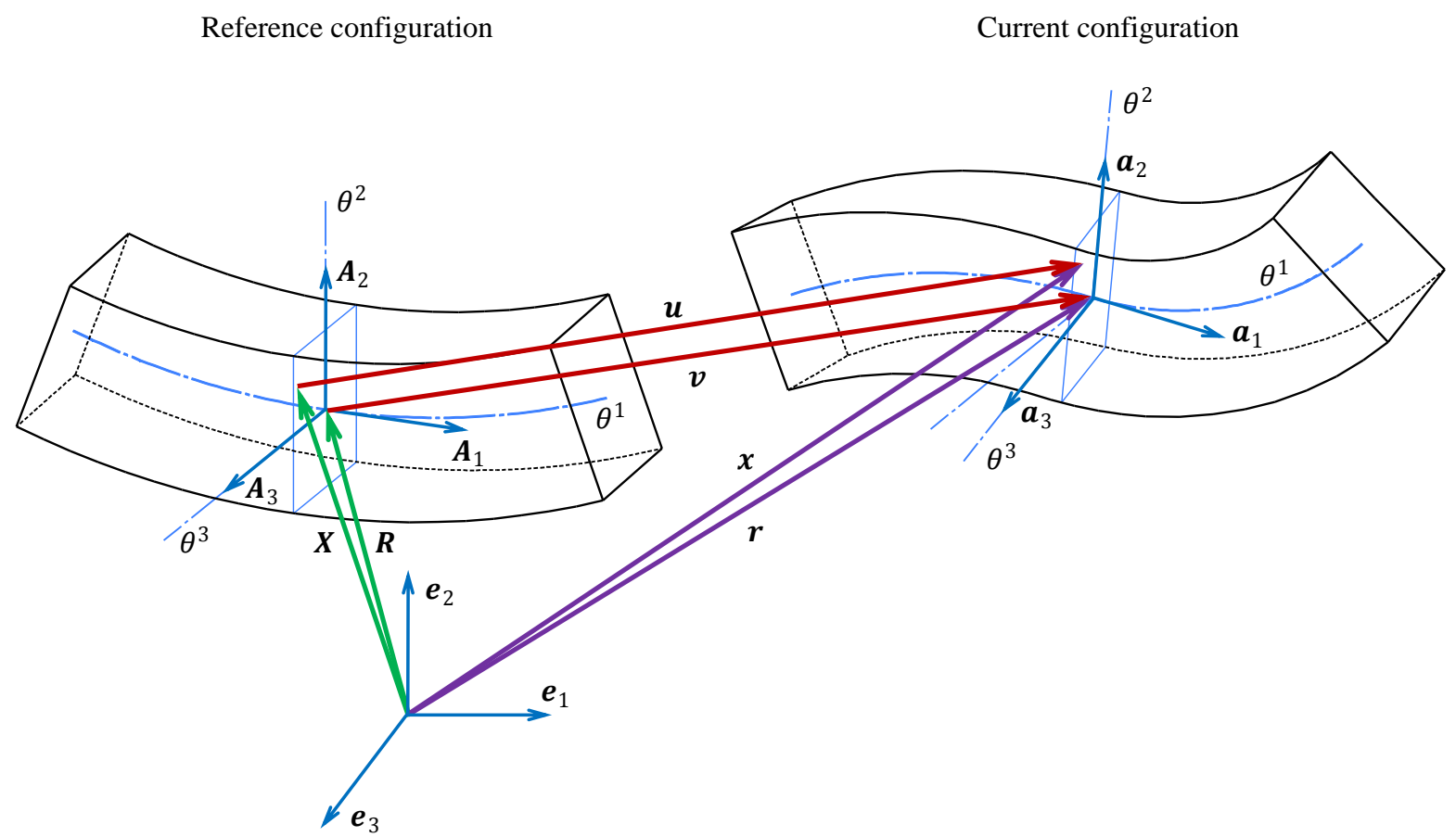

Fig. 5. Reference (undeformed) vs. current (deformed) configurations of a spatial curved beam

As shown in Fig. 5, the positions of an arbitrary point on the curve in the reference (undeformed) and current (deformed) configurations are:

$$
\begin{gathered}
\boldsymbol{X}\left(\theta^{1}, \theta^{2}, \theta^{3}\right)=\boldsymbol{R}\left(\theta^{1}\right)+\theta^{2} \boldsymbol{A}_{2}\left(\theta^{1}\right)+\theta^{3} \boldsymbol{A}_{3}\left(\theta^{1}\right) \\
\boldsymbol{x}\left(\theta^{1}, \theta^{2}, \theta^{3}\right)=\boldsymbol{r}\left(\theta^{1}\right)+\theta^{2} \boldsymbol{a}_{2}\left(\theta^{1}\right)+\theta^{3} \boldsymbol{a}_{3}\left(\theta^{1}\right)
\end{gathered}
$$

where $\theta^{i}$ are curvilinear coordinates, $\boldsymbol{r}$ and $\boldsymbol{R}$ are the position vectors of the corresponding points on the centerline and vectors $\boldsymbol{A}_{i}$ and $\boldsymbol{a}_{i}$ are the base vectors in reference and current configurations, respectively. It should be pointed out that the vectors $\boldsymbol{A}_{i}$ are related to the tangent $\mathcal{J}$, the normal $\mathcal{N}$, and the binormal $\mathcal{B}$ vectors of the geometry in its undeformed state, introduced in $\S 3.1$, as follows:

$$
\begin{gathered}
\boldsymbol{A}_{1}=\mathcal{T} \frac{\partial \xi}{\partial \theta^{1}} \\
\boldsymbol{A}_{2}=\mathcal{N} \\
\boldsymbol{A}_{3}=\mathcal{B}
\end{gathered}
$$

Using Eq. (17), the deformation vector $\boldsymbol{u}$ is then expressed as: 


$$
\boldsymbol{u}=\boldsymbol{x}-\boldsymbol{X}=(\boldsymbol{r}-\boldsymbol{R})-\theta^{2}\left(\boldsymbol{a}_{2}-\boldsymbol{A}_{2}\right)+\theta^{3}\left(\boldsymbol{a}_{3}-\boldsymbol{A}_{3}\right)
$$

or,

$$
\boldsymbol{u}=\boldsymbol{v}-\theta^{2}\left(\boldsymbol{a}_{2}-\boldsymbol{A}_{2}\right)+\theta^{3}\left(\boldsymbol{a}_{3}-\boldsymbol{A}_{3}\right)
$$

where $\boldsymbol{v}$ is the centerline displacement vector that contains the unknown DOFs. In order to express the displacement field, a two-step mapping-rotation procedure adapted from [23] is employed. This procedure first aligns the tangent vectors in deformed and undeformed configurations by applying the mapping operator $\boldsymbol{\Lambda}$, resulting in the $\boldsymbol{\Lambda} \boldsymbol{A}_{2}$ and $\boldsymbol{\Lambda} \boldsymbol{A}_{3}$ vectors, which are then rotated by the rotation matrix $\boldsymbol{Q}$ around the tangent vector to align them with $\boldsymbol{a}_{2}$ and $\boldsymbol{a}_{3}$, respectively (see Fig. 6). This mapping-rotation procedure amounts to finding $\boldsymbol{a}_{2}$ and $\boldsymbol{a}_{3}$ in the deformed state as follows (where $\phi$ is the rotational DOF):

$$
\begin{aligned}
& a_{2}=\boldsymbol{Q}(\phi) \Lambda\left(A_{1}, a_{1}\right) A_{2} \\
& a_{3}=\boldsymbol{Q}(\phi) \Lambda\left(A_{1}, a_{1}\right) A_{3}
\end{aligned}
$$

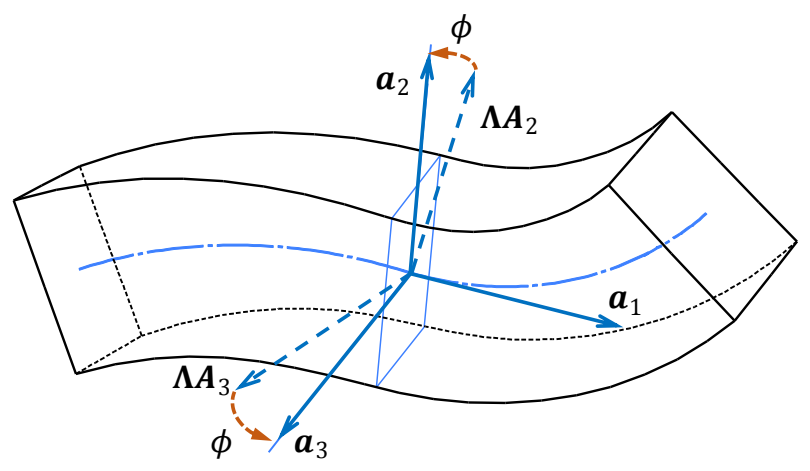

Fig. 6. Aligning base vectors in the deformed configuration by means of mapping-rotation procedure

In the above-mentioned expressions, the mapping and rotation matrices $\boldsymbol{\Lambda}$ and $\boldsymbol{Q}$ are described using the Euler-Rodriguez formula as:

$$
\begin{gathered}
\boldsymbol{\Lambda}\left(\boldsymbol{A}_{1}, \boldsymbol{a}_{1}\right)=\hat{\boldsymbol{e}} \otimes \hat{\boldsymbol{e}}+\cos (\phi)(\boldsymbol{I}-\hat{\boldsymbol{e}} \otimes \hat{\boldsymbol{e}})+\sin (\phi)(\hat{\boldsymbol{e}} \times \boldsymbol{I}) \\
\boldsymbol{Q}(\phi)=\boldsymbol{I} \cos (\phi)+\sin (\phi)\left(\boldsymbol{a}_{1} \times \boldsymbol{I}\right)
\end{gathered}
$$

where

$$
\begin{gathered}
\hat{\boldsymbol{e}}=\frac{\boldsymbol{A}_{1} \times \boldsymbol{a}_{1}}{\left\|\boldsymbol{A}_{1} \times \boldsymbol{a}_{1}\right\|} \\
\cos (\phi)=\boldsymbol{A}_{1} \cdot \boldsymbol{a}_{1} \\
\sin (\phi)=\left\|\boldsymbol{A}_{1} \times \boldsymbol{a}_{1}\right\|
\end{gathered}
$$


and $\boldsymbol{I}$ is the $3 \times 3$ identity matrix. It is to be noted that in a linear assumption, $\cos (\phi) \cong 1$ and $\sin (\phi) \cong \phi$, resulting in the following simplified forms of $\boldsymbol{\Lambda}$ and $\boldsymbol{Q}$ :

$$
\begin{gathered}
\boldsymbol{\Lambda}=\boldsymbol{I}+\left(\boldsymbol{A}_{1} \times \boldsymbol{a}_{1}\right) \times \boldsymbol{I} \\
\boldsymbol{Q}=\boldsymbol{I}+\phi\left(\boldsymbol{a}_{1} \times \boldsymbol{I}\right)
\end{gathered}
$$

In addition,

$$
a_{1}=A_{1}+u_{, 1}=A_{1}+v_{, 1}
$$

where the subscript ( ),1 refers to differentiation with respect to $\theta^{1}$. The derivatives of $\boldsymbol{a}_{2}$ and $\boldsymbol{a}_{3}$ with respect to $\theta^{1}$ will be also needed and can be obtained using the relations:

$$
\begin{aligned}
& a_{2,1}=Q_{, 1} \Lambda A_{2}+Q \Lambda_{, 1} A_{2}+Q \Lambda A_{2,1} \\
& a_{3,1}=Q_{, 1} \Lambda A_{3}+Q \Lambda_{, 1} A_{3}+Q \Lambda A_{3,1}
\end{aligned}
$$

The general nonlinear Green-Lagrange strain measure is [23]:

$$
\boldsymbol{\varepsilon}=\frac{1}{2}\left(g_{i j}-G_{i j}\right) \boldsymbol{G}^{i} \otimes \boldsymbol{G}^{j}
$$

where $\boldsymbol{G}^{i}$ are the contravariant basis vectors, $g_{i j}$ and $G_{i j}$ are the covariant components of the metric tensor in the deformed and undeformed configurations, respectively, defined by:

$$
\begin{aligned}
G_{i j} & =\boldsymbol{G}_{i} \cdot \boldsymbol{G}_{j} \\
g_{i j} & =\boldsymbol{g}_{i} \cdot \boldsymbol{g}_{j}
\end{aligned}
$$

The linearized form of $\boldsymbol{\varepsilon}$ for small displacements and strains can be determined as:

$$
\boldsymbol{\varepsilon}=\frac{1}{2}\left(\boldsymbol{u}_{, i} \cdot \boldsymbol{G}_{j}+\boldsymbol{u}_{, j} \cdot \boldsymbol{G}_{i}\right) \boldsymbol{G}^{i} \otimes \boldsymbol{G}^{j}=E_{i j} \boldsymbol{G}^{i} \otimes \boldsymbol{G}^{j}
$$

It should be noted that the covariant basis vectors in the deformed and undeformed configurations are defined as follows:

$$
\begin{gathered}
\boldsymbol{G}_{1}=\boldsymbol{X}_{, 1}=\boldsymbol{R}_{, 1}+\theta^{2} \boldsymbol{A}_{2,1}+\theta^{3} \boldsymbol{A}_{3,1} \\
\boldsymbol{G}_{2}=\boldsymbol{A}_{2} \\
\boldsymbol{G}_{3}=\boldsymbol{A}_{3} \\
\boldsymbol{g}_{1}=\boldsymbol{x}_{, 1}=\boldsymbol{a}_{1}+\theta^{2} \boldsymbol{a}_{2,1}+\theta^{3} \boldsymbol{a}_{3,1} \\
\boldsymbol{g}_{2}=\boldsymbol{a}_{2} \\
\boldsymbol{g}_{3}=\boldsymbol{a}_{3}
\end{gathered}
$$

Using Eqs.(26) to (31), all non-zero terms of strain tensor are calculated as follows: 


$$
\begin{aligned}
& E_{11}=\boldsymbol{u}_{, 1} \cdot \boldsymbol{G}_{1}=\overbrace{\boldsymbol{v}_{, 1} \cdot \boldsymbol{A}_{1}}^{I_{1}^{11}}+\theta^{2} \overbrace{\left(\boldsymbol{a}_{2,1} \cdot \boldsymbol{A}_{1}-\boldsymbol{A}_{2,1} \cdot \boldsymbol{A}_{1}+\boldsymbol{v}_{, 1} \cdot \boldsymbol{A}_{2,1}\right)}^{I_{2}^{11}} \\
& +\theta^{3} \overbrace{\left(\boldsymbol{a}_{3,1} \cdot \boldsymbol{A}_{1}-\boldsymbol{A}_{3,1} \cdot \boldsymbol{A}_{1}+\boldsymbol{v}_{, 1} \cdot \boldsymbol{A}_{3,1}\right)}^{I_{3}^{11}}=I_{1}^{11}+\theta^{2} I_{2}^{11}+\theta^{3} I_{3}^{11} \\
& E_{12}=\frac{1}{2}\left(\boldsymbol{u}_{, 1} \cdot \boldsymbol{G}_{2}+\boldsymbol{u}_{, 2} \cdot \boldsymbol{G}_{1}\right) \\
& =\frac{1}{2}[\overbrace{\left(\boldsymbol{v}_{, 1} \cdot \boldsymbol{A}_{2}+\boldsymbol{a}_{2} \cdot \boldsymbol{A}_{1}\right)}^{I_{1}^{12}}+\theta^{2} \overbrace{\left(\boldsymbol{a}_{2,1} \cdot \boldsymbol{A}_{2}+\boldsymbol{a}_{2} \cdot \boldsymbol{A}_{2,1}\right)}^{I_{2}^{12}} \\
& +\theta^{3} \overbrace{\left(\boldsymbol{a}_{3,1} \cdot \boldsymbol{A}_{2}-\boldsymbol{A}_{3,1} \cdot \boldsymbol{A}_{2}+\boldsymbol{a}_{2} \cdot \boldsymbol{A}_{3,1}-\boldsymbol{A}_{2} \cdot \boldsymbol{A}_{3,1}\right)}^{I_{3}^{12}}]=I_{1}^{12}+\theta^{2} I_{2}^{12}+\theta^{3} I_{3}^{12} \\
& E_{13}=\frac{1}{2}\left(\boldsymbol{u}_{, 1} \cdot \boldsymbol{G}_{3}+\boldsymbol{u}_{, 3} \cdot \boldsymbol{G}_{1}\right) \\
& =\frac{1}{2}[\overbrace{\left(\boldsymbol{v}_{, 1} \cdot \boldsymbol{A}_{3}+\boldsymbol{a}_{3} \cdot \boldsymbol{A}_{1}\right)}^{I_{1}^{13}}+\theta^{2} \overbrace{\left(\boldsymbol{a}_{2,1} \cdot \boldsymbol{A}_{3}-\boldsymbol{A}_{2,1} \cdot \boldsymbol{A}_{3}+\boldsymbol{a}_{3} \cdot \boldsymbol{A}_{2,1}-\boldsymbol{A}_{3} \cdot \boldsymbol{A}_{2,1}\right)}^{I_{2}^{13}} \\
& +\theta^{3} \overbrace{\left(\boldsymbol{a}_{3,1} \cdot \boldsymbol{A}_{3}+\boldsymbol{a}_{3} \cdot \boldsymbol{A}_{3,1}\right)}^{I_{3}^{13}}]=I_{1}^{13}+\theta^{2} I_{2}^{13}+\theta^{3} I_{3}^{13}
\end{aligned}
$$

In deriving the above equations, the following assumptions have been made:

- For slender beams, quadratic terms including $\left(\theta_{2}\right)^{2},\left(\theta_{3}\right)^{2}$ and $\theta_{2} \theta_{3}$ are neglected.

- Since, in Euler-Bernoulli theory, the cross-section remains normal to the centerline and crosssectional dimensions remain unchanged, one obtains:

$$
\begin{gathered}
\boldsymbol{A}_{1} \cdot \boldsymbol{A}_{2}=0 ; \quad \boldsymbol{A}_{1} \cdot \boldsymbol{A}_{3}=0 ; \quad \boldsymbol{a}_{1} \cdot \boldsymbol{a}_{2}=0 ; \quad \boldsymbol{a}_{1} \cdot \boldsymbol{a}_{3}=0 \\
\boldsymbol{A}_{2,1} \cdot \boldsymbol{A}_{2}=0 ; \quad \boldsymbol{A}_{3,1} \cdot \boldsymbol{A}_{3}=0
\end{gathered}
$$

- According to Euler-Bernoulli theory, we also have:

$$
\begin{gathered}
E_{22}=E_{33}=E_{23}=0 \\
I_{1}^{12}=I_{2}^{12}=I_{1}^{13}=I_{2}^{13}=0
\end{gathered}
$$

- In the computation of $I_{1}^{11}, I_{2}^{11}, I_{3}^{11}, I_{3}^{12}$ and $I_{2}^{13}$, all nonlinear terms containing more than one DOF (or their derivatives) are neglected.

- The Euler-Bernoulli beam theory necessitates having at least $C^{1}$ continuity throughout the curve and therefore a quadratic approximation should be at least employed. However, in order to avoid membrane locking (i.e., incapability of reflecting inextensible bending), B-spline approximations of degree three or higher will be adopted in the following (see, e.g., [49, 50]). 
With the strain and deflection components of Eqs. (32) to (34), and using Hamilton's principle, one can obtain:

$$
\delta U-\delta T-\delta W=0
$$

where $U$ is the internal potential energy, $T$ is the kinetic energy, and $W$ is the work done by the external forces, such that:

$$
\begin{gathered}
\delta U=\int_{\Omega} \delta\left(\boldsymbol{\varepsilon}^{T}\right) \boldsymbol{C} \boldsymbol{\varepsilon} d \Omega \\
\delta T=\int_{\Omega} \delta\left(\dot{\boldsymbol{u}}^{T}\right) \rho \dot{\boldsymbol{u}} d \Omega \\
\delta W=\int_{\Omega} \delta \boldsymbol{u}^{T} \boldsymbol{f}_{b} d \Omega+\int_{A} \delta \boldsymbol{u}^{T} \boldsymbol{f}_{S} d A
\end{gathered}
$$

In the above equations, the matrix $\boldsymbol{C}$ describes the material properties and is defined as:

$$
\boldsymbol{C}=\left[\begin{array}{lll}
E & 0 & 0 \\
0 & G & 0 \\
0 & 0 & G
\end{array}\right]
$$

being $E, G$ and $\rho$ the values of elasticity modulus, shear modulus, and density, respectively. In addition, $\boldsymbol{f}_{b}$ and $\boldsymbol{f}_{S}$ are the body and surface loads, respectively.

\subsection{Isogeometric Discretization}

Based on the isoparametric concept of the IGA approach, the discrete displacement field of the centerline $(v)$ and the rotational DOF $(\phi)$ are defined by B-spline shape functions representing the beam geometry. Referring to Fig. 7, within the $k$-th element $\Omega^{e}: s \in\left[s_{k-1}, s_{k}\right]$ on the curved beam that is equivalent to $\xi \in\left[\xi_{p+k-1}, \xi_{p+k}\right]$ on the parameter space (in the case of non-repetitive internal knots), the local support property of B-spline curves necessitates having $p+1$ non-zero (i.e., active) $C^{p-1}$ basis functions, namely $N_{k-1}^{p}(\xi)$ to $N_{k+p-1}^{p}(\xi)$ where the corresponding control points are $\boldsymbol{P}^{k-1}$ to $\boldsymbol{P}^{k+p-1}$ [38]. Table 1 presents the geometry and deformation fields of spatial curved beams in the isogeometric framework where $x, y$ and $Z$ denote the position of an arbitrary point on the centerline, $\left[X^{i}, Y^{i}, Z^{i}\right]$ is the position of $i$-th control point of the geometry in space, $v_{x}^{e}, v_{y}^{e}, v_{z}^{e}$ and $\phi^{e}$ are DOFs at the element level, and $V_{x}^{i}, V_{y}^{i}, V_{z}^{i}$ and $\Phi^{i}$ are their respective control variables. Finally, it should be also pointed out that integration over the element space is performed in the parent element domain $\tilde{\xi} \in[-1,1]$ using a standard Gauss-Legendre quadrature rule. 


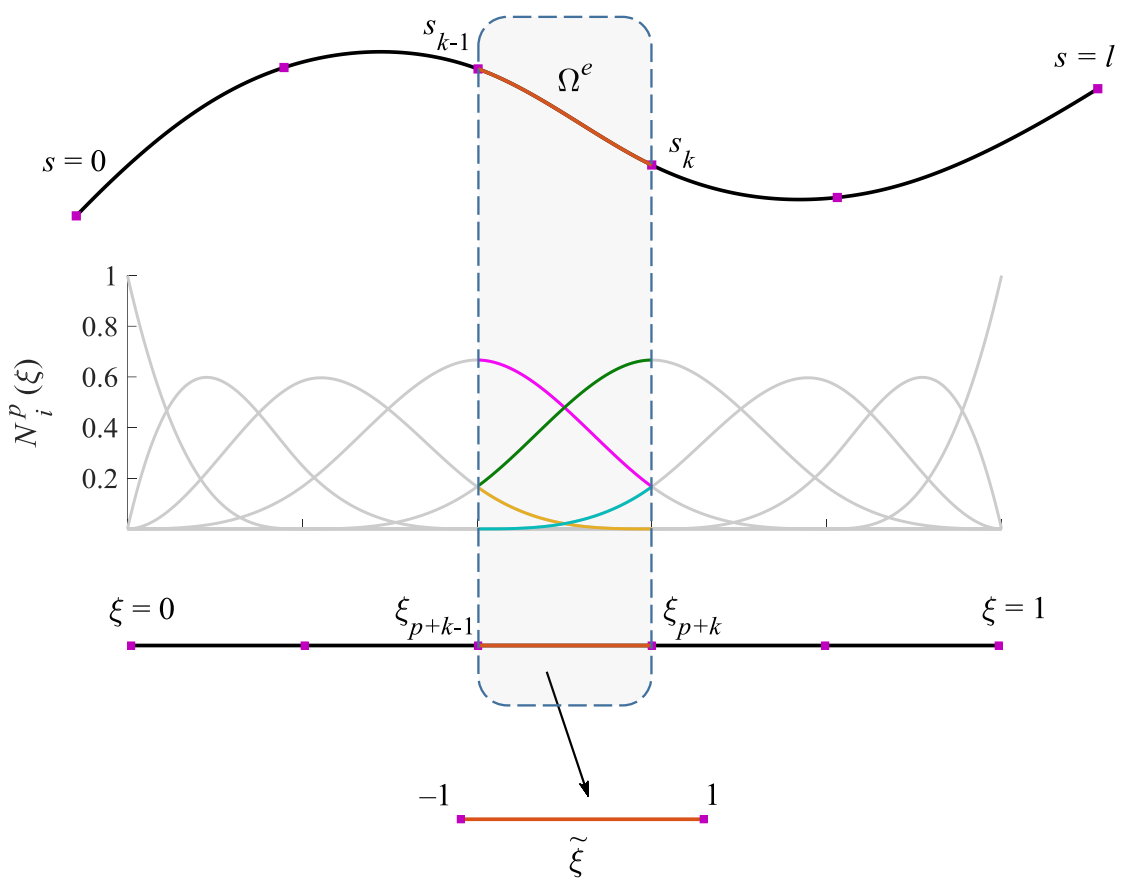

Geometric domain

Basis functions

Parametric domain

Parent element

Fig. 7. Characterization of a curved beam element in IGA

Table 1. B-spline representation of the geometry and field variables of spatial curved beams

\begin{aligned} \hline Geometry & Field variables over the $k$-th element \\ \hline$x(\xi)=\sum_{i=0}^{n} N_{i}^{p}(\xi) X^{i} & v_{x}^{e}(\xi)=\sum_{i=k-1}^{k+p-1} N_{i}^{p}(\xi) V_{x}^{i} \\ y(\xi)=\sum_{i=0}^{n} N_{i}^{p}(\xi) Y^{i} & v_{y}^{e}(\xi)=\sum_{i=k-1}^{k+p-1} N_{i}^{p}(\xi) V_{y}^{i} \\ z(\xi)=\sum_{i=0}^{n} N_{i}^{p}(\xi) Z^{i} & v_{z}^{e}(\xi)=\sum_{i=k-1}^{k+p-1} N_{i}^{p}(\xi) V_{z}^{i} \\ \phi^{e}(\xi) & =\sum_{i=k-1}^{k+p-1} N_{i}^{p}(\xi) \Phi^{i}\end{aligned}$

By implementing the isogeometric framework, the stiffness and mass matrices and the force vector at the element level are obtained through the discretization of Eq. (37). The above-mentioned matrices are then assembled to solve the following static and eigenvalue problems (see, e.g., [40, 50, 51]): 


$$
\begin{gathered}
\boldsymbol{K} \boldsymbol{q}=\boldsymbol{F} \\
\left(\boldsymbol{K}-\omega^{2} \boldsymbol{M}\right) \boldsymbol{q}=\mathbf{0}
\end{gathered}
$$

where $\boldsymbol{K}, \boldsymbol{M}$ and $\boldsymbol{F}$ are the global stiffness matrix, the global mass matrix, and the global force vector, respectively, while $\omega$ are the eigenfrequencies and $\boldsymbol{q}$ is the vector of nodal degrees of freedom, i.e., $\boldsymbol{q}=\left[V_{x}^{0}, \cdots, V_{x}^{n}, V_{y}^{0}, \cdots, V_{y}^{n}, V_{z}^{0}, \cdots, V_{z}^{n}, \Phi^{0}, \cdots, \Phi^{n}\right]^{T}$. Given the nodal DOFs, one can simply obtain the distribution of stress results (like bending moments) along the beam using the derivatives of the displacement fields.

\section{Case Studies}

The different knot placement techniques are herein tested by three case studies of free-form geometries, namely the Tschirnhausen, the elliptic spiral, and the Lissajous beams. For this purpose, the natural frequencies and static deflection under an end force are obtained for the mentioned beams using isogeometric analysis in combination with different knot placement techniques. Some explanatory remarks regarding the numerical results of this section follow:

- In all examples, a circular cross-section of $0.1 \mathrm{~m}$ radius is assumed. In addition, the Young modulus, the Poisson ratio, and the density of all curved beams are assumed to be $E=200 \mathrm{GPa}$, $v=0.3$, and $\rho=1000 \mathrm{~kg} / \mathrm{m}^{3}$, respectively, while the shear modulus is calculated as $G=\frac{E}{2(1+v)}$.

- The geometry of each example is constructed by B-spline curves of degrees 3, 4, and 5 with arbitrary input data points. The parameterization is based on the chord-length approach (see \$2.2) and the number of control points is selected in such a way that a desirable curve fit as well as converged IGA results are achieved.

- Increasing the number of control points at each refinement level needs a new curve fitting process, so that the beam geometry will be subject to a slight change (i.e., improved in terms of fitting error). As a result, the convergence rates may be different as compared to typical $h-, p$ - and $k$-refinements (see §5.3).

- Since an analytical solution does not exist for the considered curved beam examples, IGA results are compared with "overkill" finite element results, obtained with the commercial software ABAQUS by generating appropriate meshes of quadratic beam elements at least 100 times finer than the finest adopted IGA mesh. As a result, theoretical convergence rates of deflection and frequency errors cannot be determined.

- For different natural frequencies, the convergence behavior might be different. The characteristics of the basis functions and the positions of knots (obtained through data fitting process) with respect to different mode shapes are our main justification for such a trend.

- In particular, the reference results for the first three natural modes are computed and listed in Table 2. 
Table 2. Reference finite element results for the first three natural frequencies of the case studies (Hz)

\begin{tabular}{lccc}
\hline & Mode 1 & Mode 2 & Mode 3 \\
\hline The Tschirnhausen beam & 3.644 & 3.879 & 18.678 \\
The elliptic spiral beam & 0.770 & 0.786 & 0.995 \\
The Lissajous beam & 9.172 & 9.823 & 14.3108 \\
\hline
\end{tabular}

\subsection{The Tschirnhausen Beam}

The first example is the Tschirnhausen curved beam, whose initial geometry is planar. Input data points of the curve are obtained using the following analytical formula:

$$
\begin{aligned}
& x=-3\left(t^{2}-3\right) \\
& y=-t\left(t^{2}-3\right)
\end{aligned}
$$

where 1000 input data points are considered for the current analysis (a selection of these points is depicted in Fig. 8). The curve is clamped at the right end and is subjected to a $2 \mathrm{KN}$ point load in the $z$-direction at the left end.

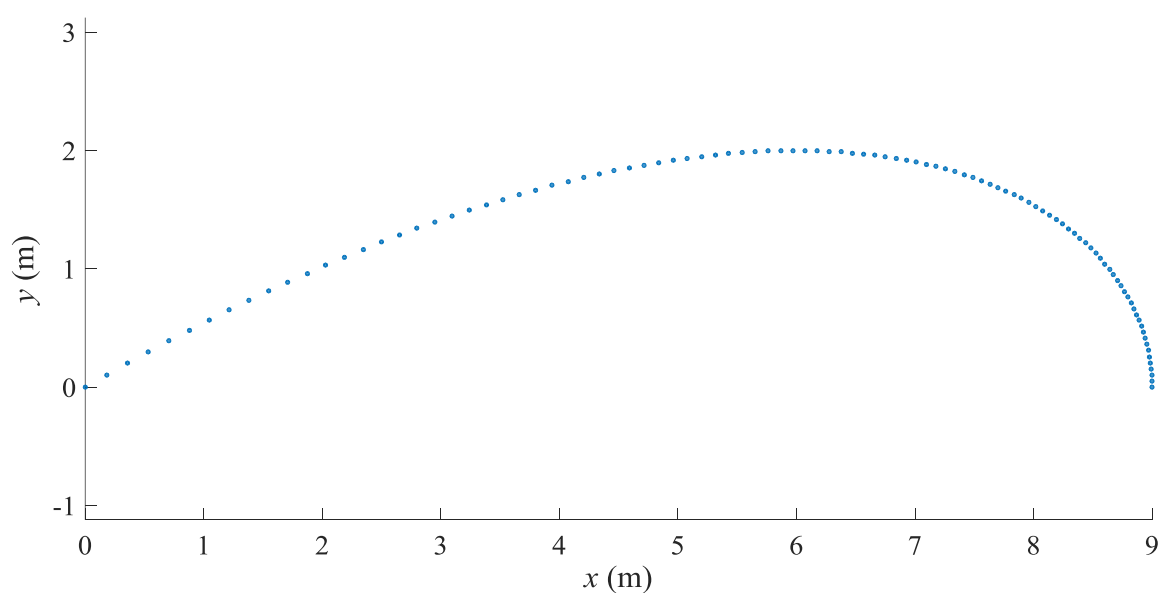

Fig. 8. Input data points for the Tschirnhausen beam

In order to construct a suitable geometry for IGA, B-spline curves with different numbers of control points are fitted. In order to explore the effect of different knot placement techniques on the shape functions of the field variables in IGA, the cubic basis function plots and respective knot positions are depicted in Fig. 9 for a coarse mesh with 10 control points. As it can be seen, the generated knot vectors and the values of basis functions are dependent on the positions of input dataset when the De Boor's and Piegl and Tiller's algorithms are employed. 


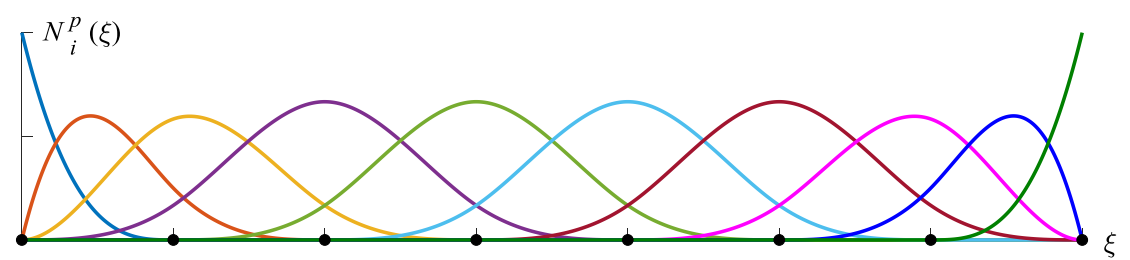

(a)

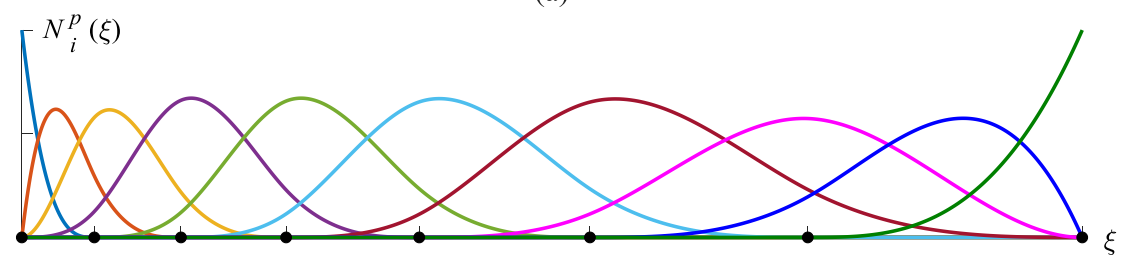

(b)

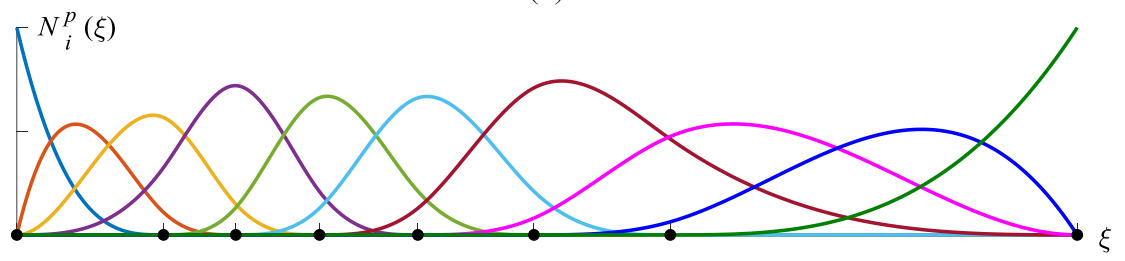

(c)

Fig. 9. Cubic basis function plots and respective knot positions for a coarse mesh (with 10 control points) of the Tschirnhausen beam: (a) uniform knot placement, (b) De Boor's algorithm, (c) Piegl and Tiller's algorithm

The $L^{2}$-norm error of the deflection $\left\|v-v_{\text {FEA }}\right\|_{L^{2}}$ and the frequency error $\left|\omega / \omega_{\text {FEA }}-1\right|$ of the first three natural modes versus the number of approximating control points are compared for different knot placement techniques and different degrees of basis functions in Fig. 10 (the errors are computed with reference to the "overkill" finite element results). It can be seen in the figure that convergence plots reach a plateau in the 2nd and 3rd modes, while, in the 1st mode or in the static deflection, more control points are needed to reach convergence and a lower convergence rate is observed. This might be due to the fact that in these cases the planar beam undergoes an out-of-plane deformation creating some shear strain that cannot be fully captured by the Euler-Bernoulli beam theory. The in-plane and out-of-plane mode shapes of this example are illustrated in Fig. 11. The results show that the use of the De Boor's and Piegl and Tiller's knot placement algorithms leads to more accurate outputs, especially when a coarse mesh is employed, while the uniform knot placement method is the least effective. One reason for the effectiveness of the De Boor's algorithm lies within the fact that it generates more accurate geometries as shown quantitatively in Fig. 12 through the least-squares fitting error. Nevertheless, it is not always true that less approximation errors lead to IGA results with higher accuracies. For example, in this case study, the results of the De Boor's algorithm for quintic basis functions are less accurate compared to Piegl and Tiller's algorithm (Fig. 10), while in terms of fitting error (Fig. 12), the De Boor's algorithm is more accurate for the same number of control points. It is important to note that in computer-aided design, the convergence rate of curve fitting problems depends on different items. For instance, the number and distribution of input data points, the degree of the basis functions, the parameter selection and knot placement schemes, and even the complexity of the curve (i.e., how curvature and torsion vary throughout the curve) can influence the convergence rate (see, e.g., [38]), as it can be also observed in the presented results. Therefore, one can hardly predict a theoretical convergence rate for curve fitting problems. 

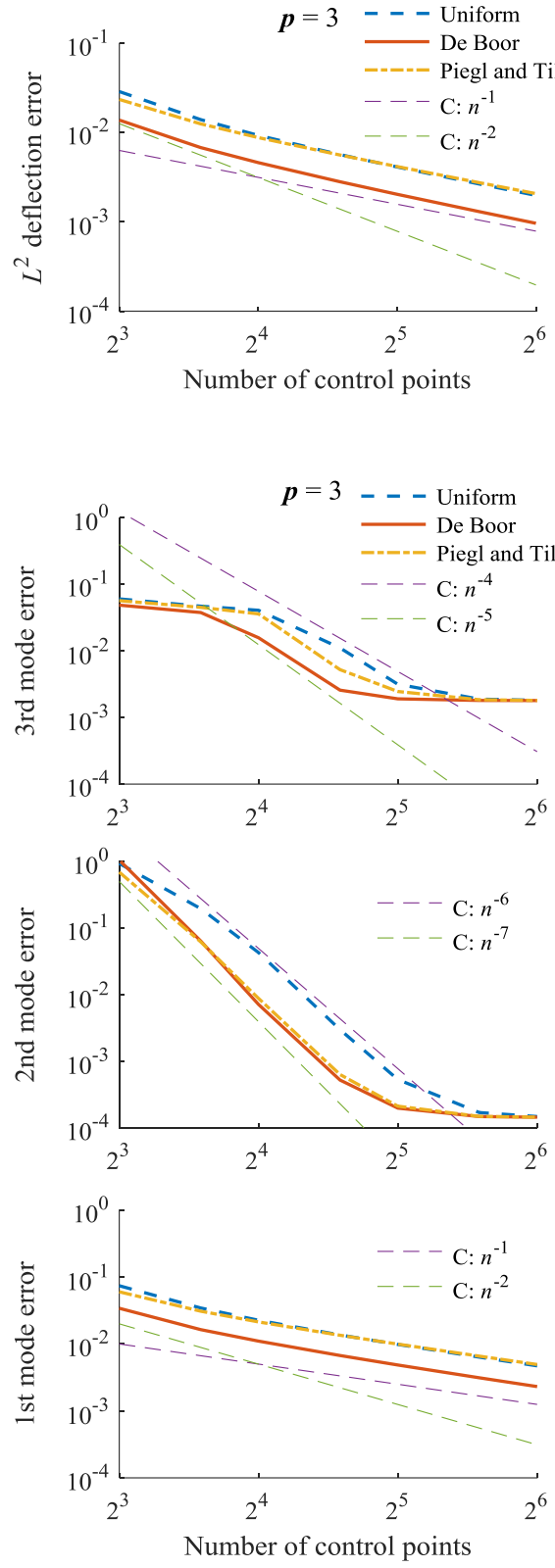

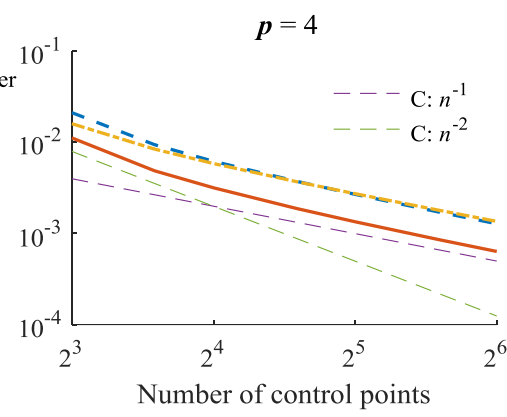

(a)
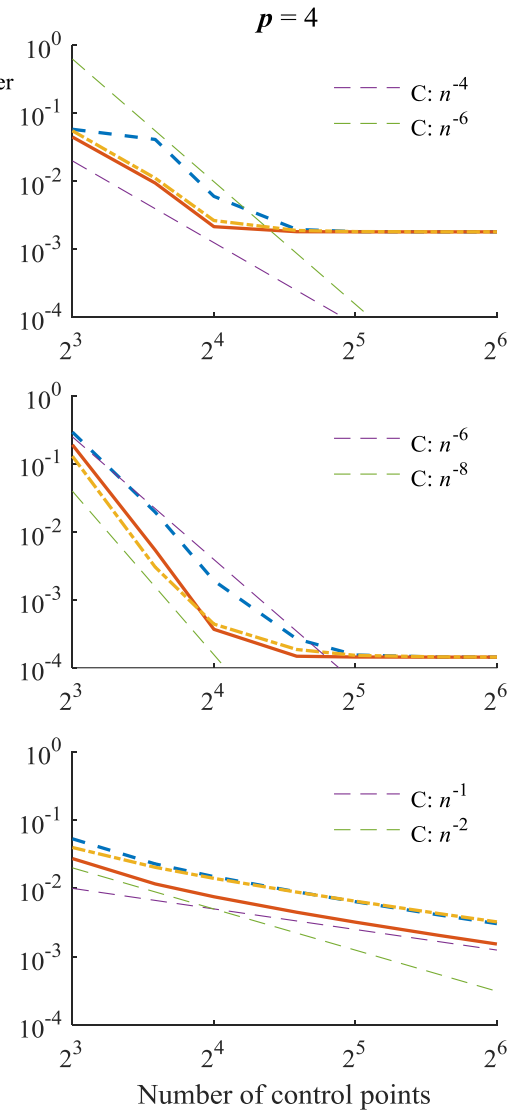

(b)

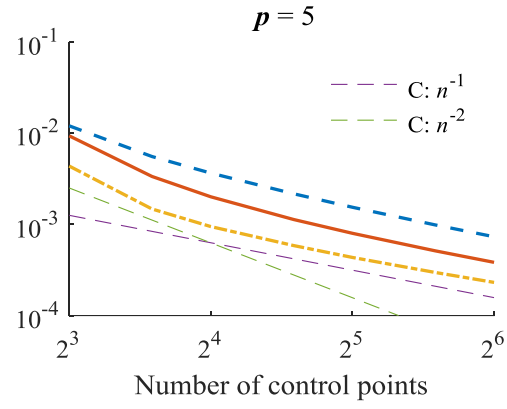

$p=5$
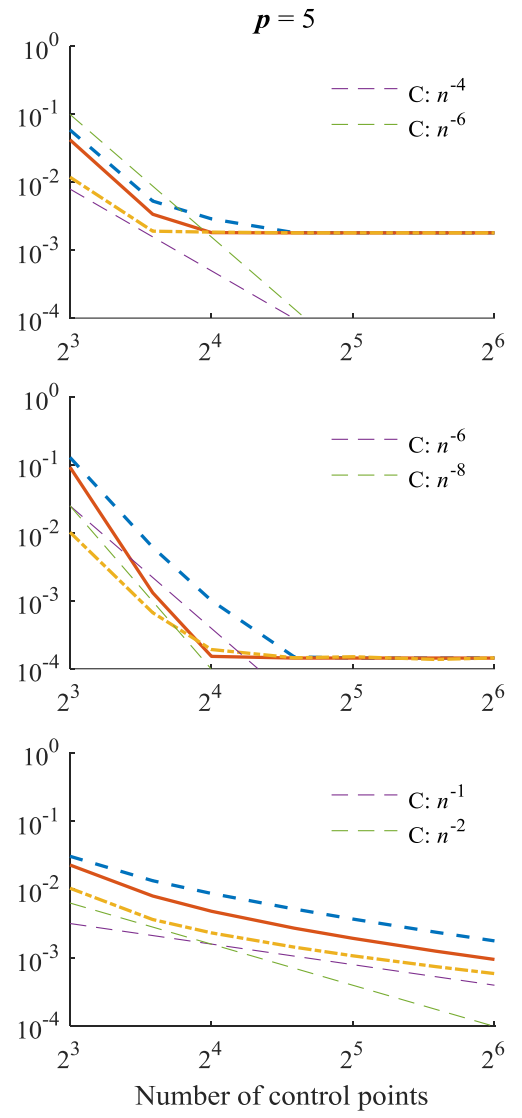

Fig. 10. Comparison of different knot placement techniques in (a) static deflection and (b) natural frequency results for the Tschirnhausen beam: computation error vs. number of approximating control points for different spline degrees

The entire frequency spectra of a coarse and a relatively fine considered mesh (of 10 and 22 control points, respectively) are also illustrated in Fig. 13 for different knot placement approaches. In this figure, the resulting natural frequencies $\omega$ are normalized with respect to the reference FEA solution, $\omega_{\mathrm{FEA}}$, and plotted versus the mode number, $i$, normalized with respect to the total number of DOFs, $N$. The frequency spectra graphs show that IGA results obtained by De Boor's knot placement algorithm are more reliable in lower natural frequencies and also when a coarse mesh is considered. In fact, there is no distinguishable difference among the three studied knot placement techniques for higher frequencies. 


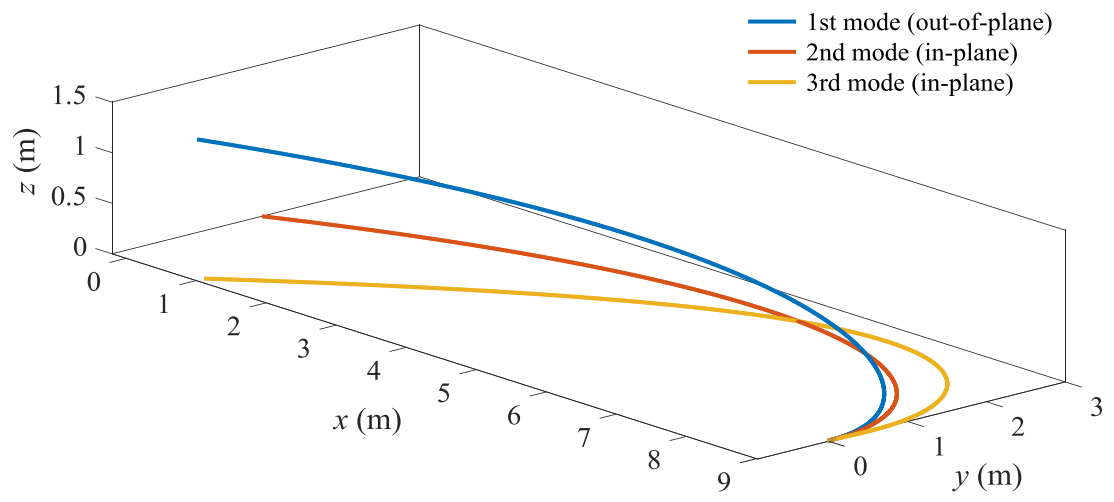

Fig. 11. First three natural modes for the Tschirnhausen beam
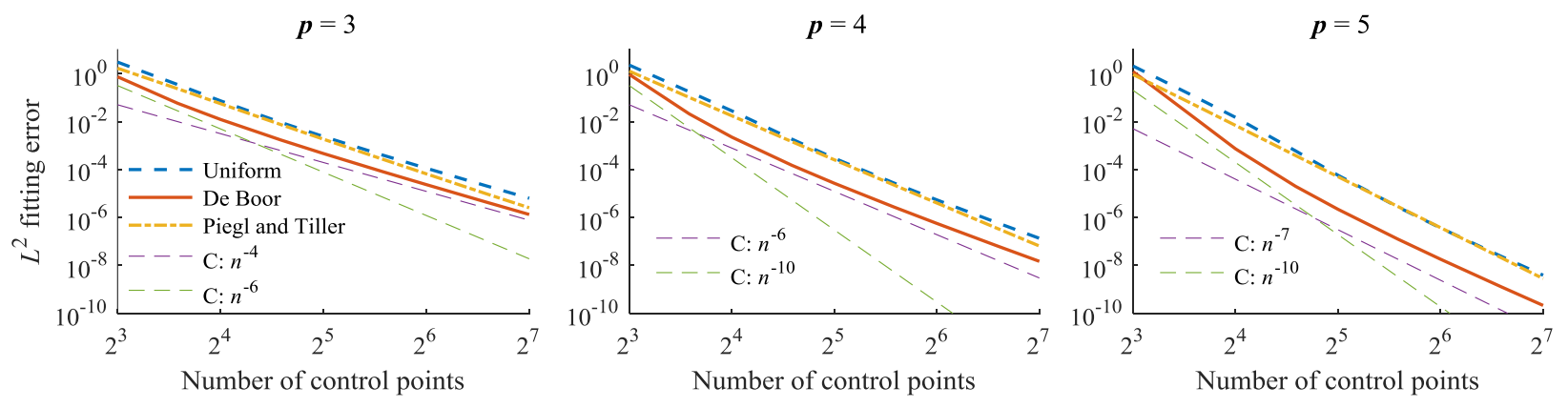

Fig. 12. Least-squares fitting error for the Tschirnhausen beam vs. number of approximating control points for different knot placement techniques and different spline degrees

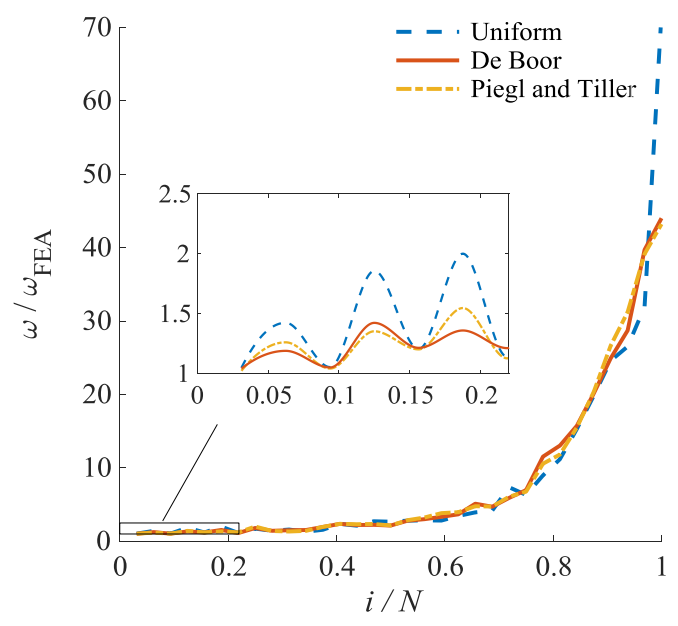

(a)

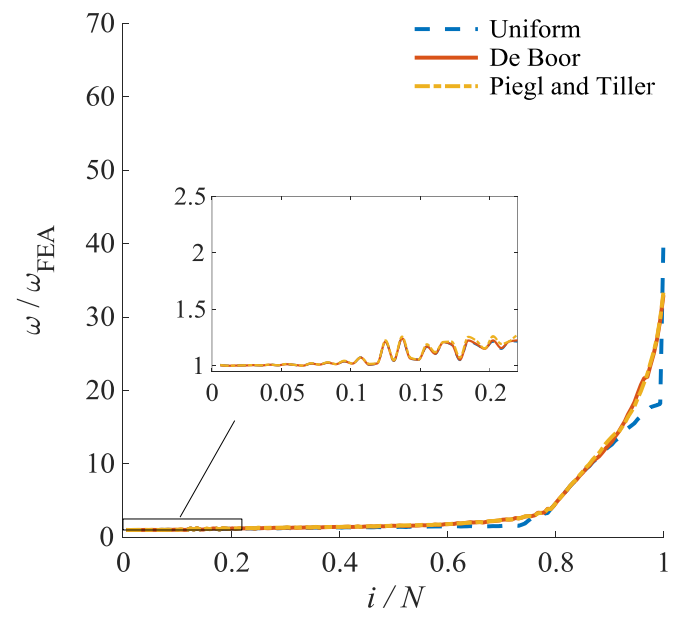

(b)

Fig. 13. Frequency spectra for the Tschirnhausen beam for different knot placement techniques: (a) a coarse mesh with 10 control points (i.e., $40 \mathrm{DOFs}$ ), (b) a relatively fine mesh with 22 control points (i.e., $88 \mathrm{DOFs})$ 
Finally, considering that engineers are always interested in a good approximation of stress resultants like bending moments, the relative $L^{2}$-norm errors of bending moments for the Tschirnhausen beam, obtained by cubic basis functions and different knot placement algorithms, are shown in Fig. 14.

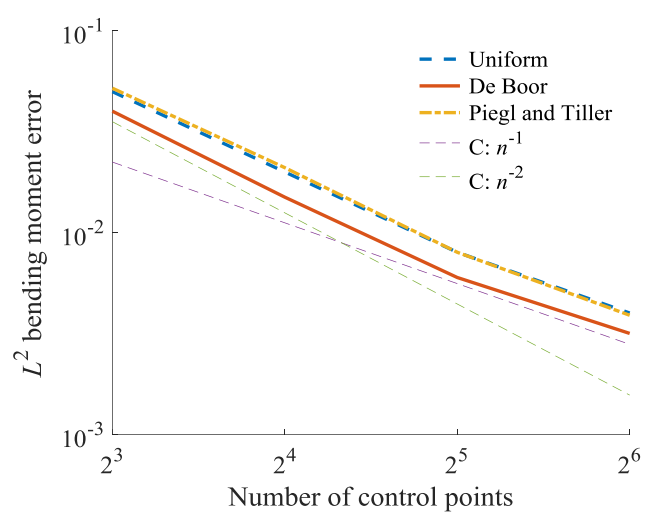

Fig. 14. Relative $L^{2}$-norm errors of bending moment resultants throughout the Tschirnhausen beam with cubic basis

\subsection{The Elliptic Spiral Beam}

An elliptic spiral curve is considered as the second example. Spirals are well-known spatial geometries that can be frequently seen in common engineering structures. The elliptic spiral of the current case study has variable curvature and torsion since its base curve is an ellipse. Some input data points of this example are shown in Fig. 15 noting that the structure is assumed to be clamped at the lower end and undergoes a tip force of $2 \mathrm{KN}$ in the $z$-direction at the upper end. The analytical expression of the curve is represented in Eq. (42). It should be noticed that the elliptic spiral has an exact NURBS representation as described in [29]. However, if the NURBS curve fitting is of interest, a nonlinear optimization problem should be solved to find unknown control points and respective weights (see, e.g., [42, 43]). This nonlinear optimization procedure is relatively expensive for IGA compared to the currently investigated B-spline curve approximation taking into account that we can reach a significant accuracy in results with more control points and by employing appropriate parameterization and knot placement schemes.

$$
\begin{gathered}
x=2 \cos (t) \\
y=4 \sin (t) \\
z=\frac{t}{4}
\end{gathered}
$$




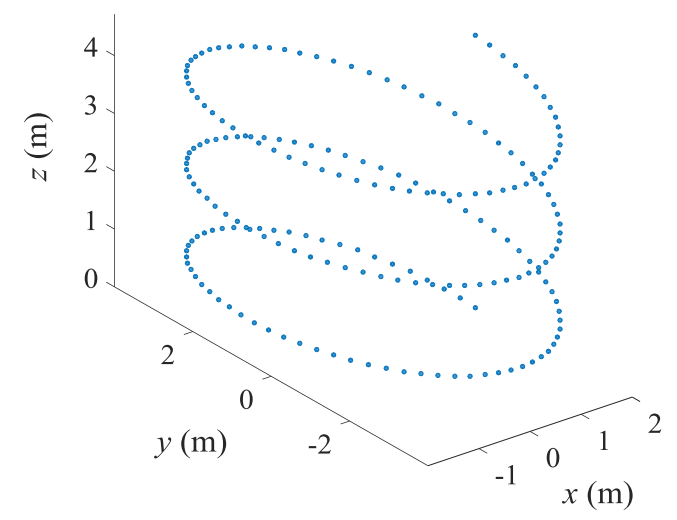

Fig. 15. Input data points of the elliptic spiral beam

The effect of different knot placement techniques on the cubic basis functions and respective knot positions of the elliptic spiral example are depicted in Fig. 16 for a coarse mesh with 50 control points.

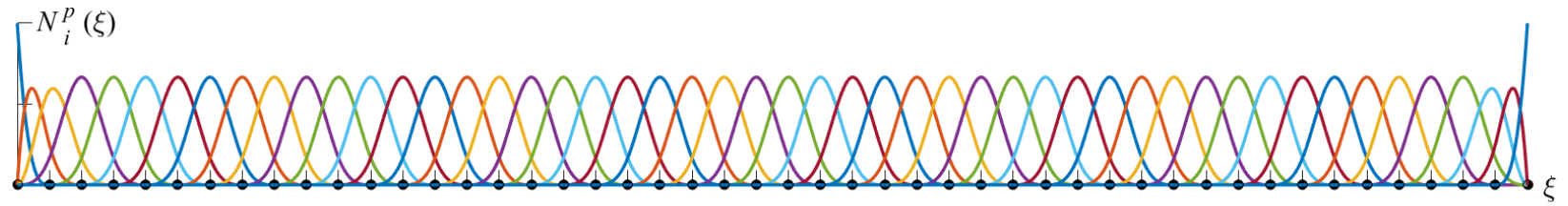

(a)

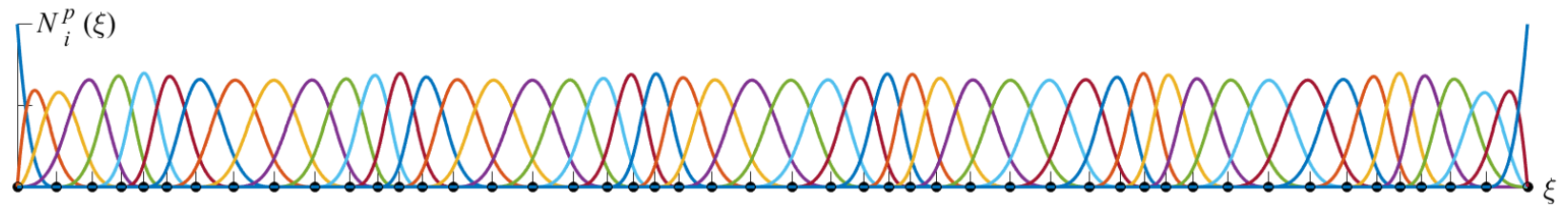

(b)

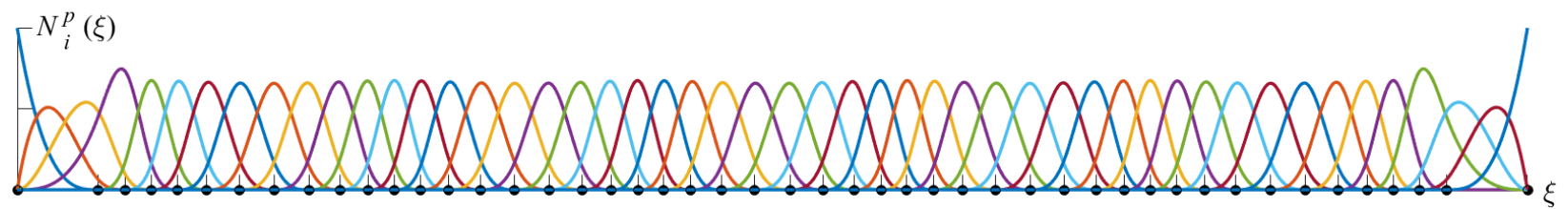

(c)

Fig. 16. Cubic basis function plots and respective knot positions for a coarse mesh (with 50 control points) of the elliptic spiral beam: (a) uniform knot placement, (b) De Boor's algorithm, (c) Piegl and Tiller's algorithm

The comparisons of the IGA results obtained by different knot placement techniques are presented in Fig. 17a and Fig. 17b for the static deflection and the first three natural frequencies, respectively. The leastsquares fitting error is also shown in Fig. 18. The results show again the superiority of the De Boor's algorithm since it typically leads to more accurate geometries as well as more accurate IGA results (although the Piegl and Tiller's algorithm may be occasionally better). We also highlight the poor performance of the uniform knot placement strategy. The entire frequency spectra are also depicted in 
Fig. 19. The full spectra plots inform us that in higher frequencies, all knot vector generation methods result in almost the same accuracy. Nevertheless, in lower frequencies and for coarse meshes, the uniform knot placement technique shows again poor results. Finally, the relative $L^{2}$-norm errors of bending moments for the elliptic spiral beam, obtained by cubic basis functions and different knot placement algorithms, are shown in Fig. 20.
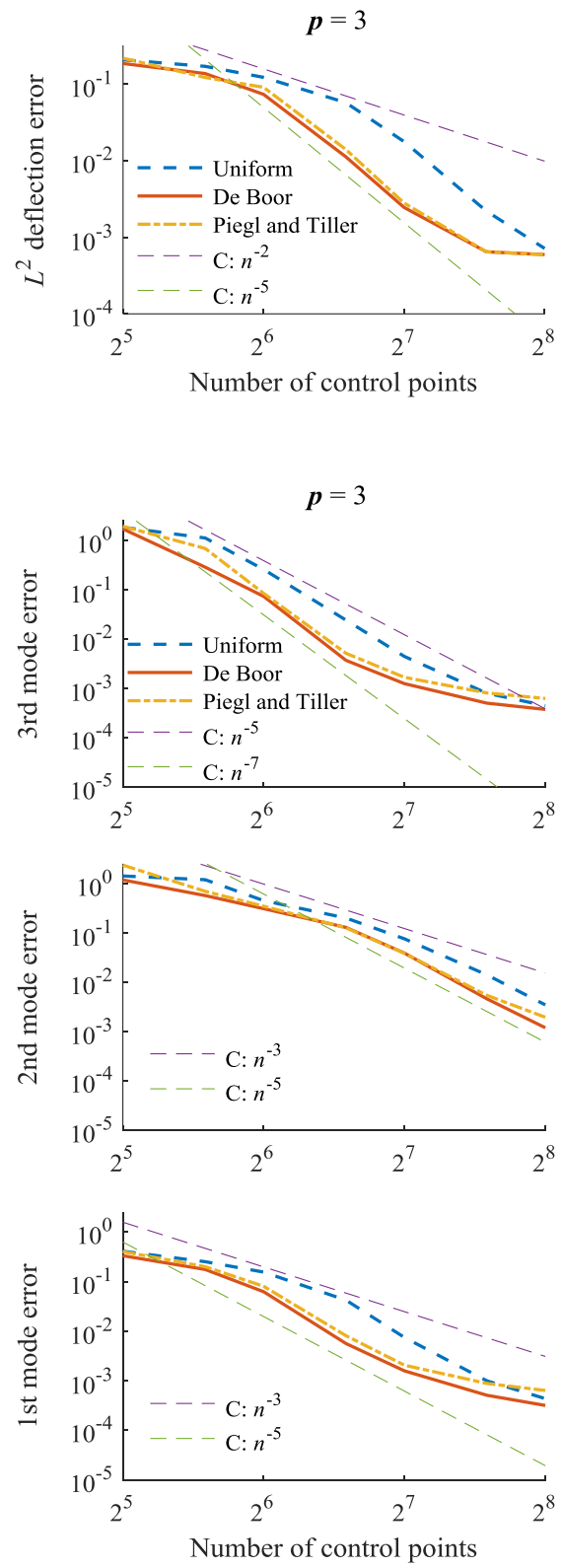

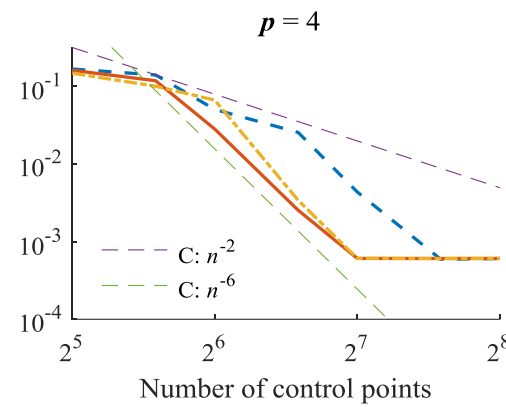

(a)
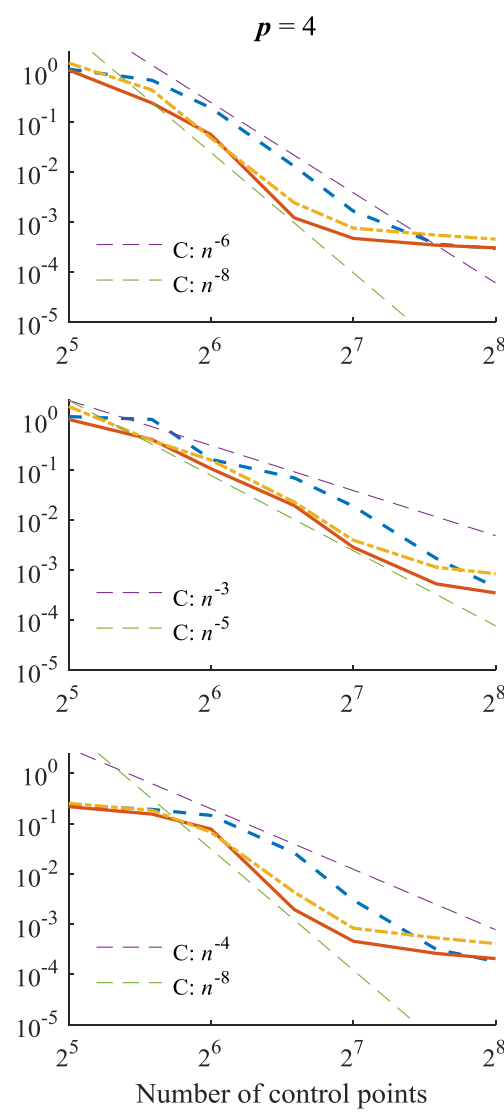

(b)
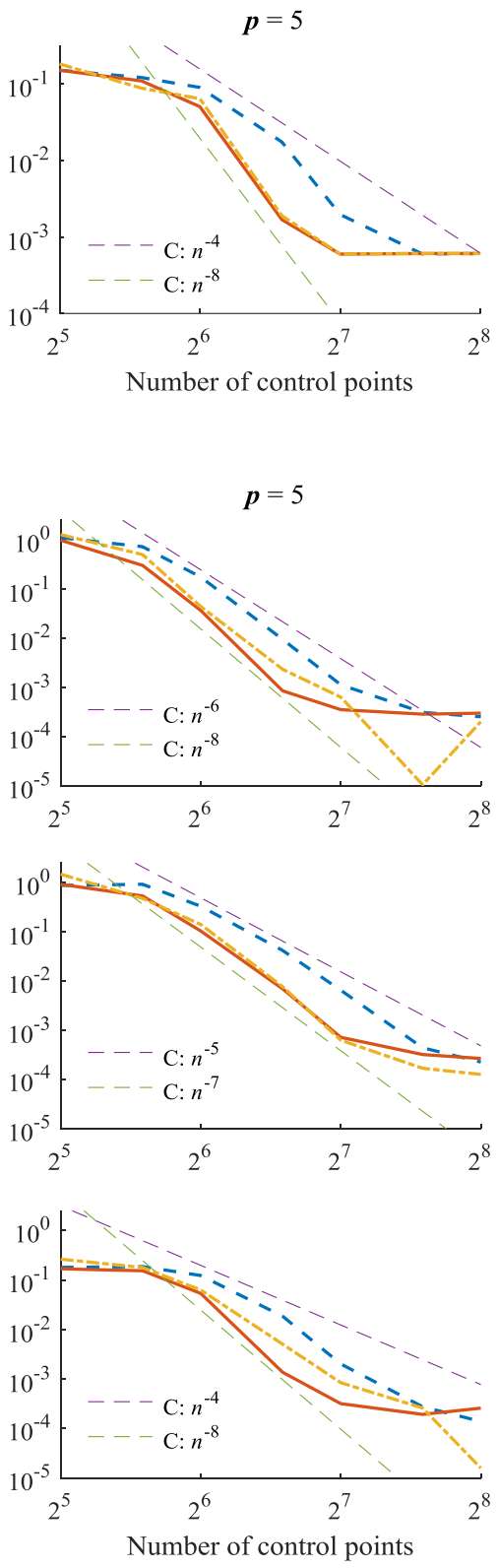

Fig. 17. Comparison of different knot placement techniques in (a) structural static and (b) natural frequency analyses of the elliptic spiral beam: computation error vs. number of approximating control points for different spline degrees 

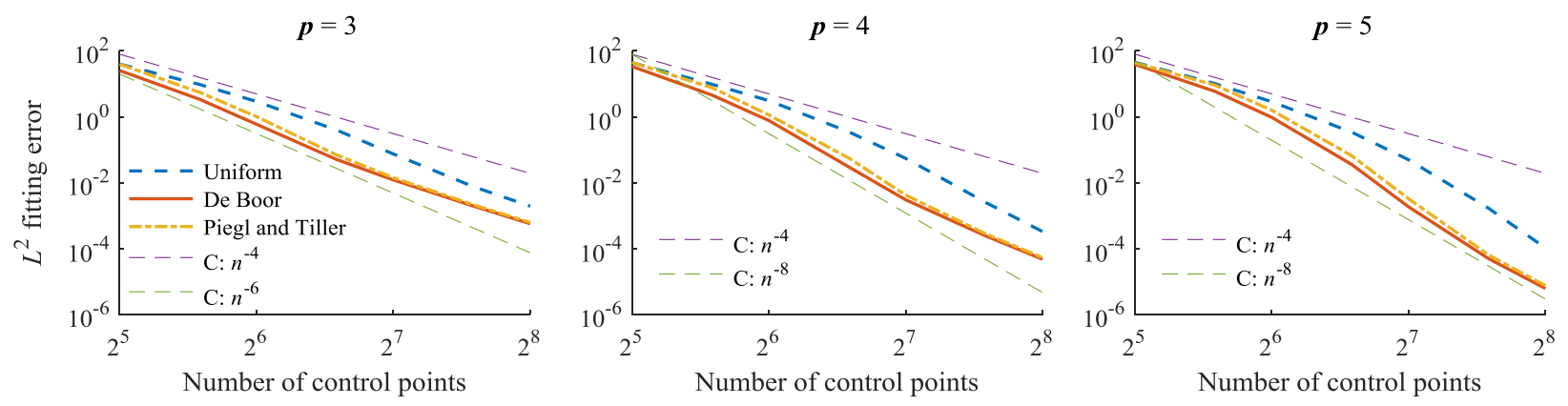

Fig. 18. Least-squares fitting error of the elliptic spiral beam vs. number of approximating control points for different knot placement techniques and different degrees of basis functions

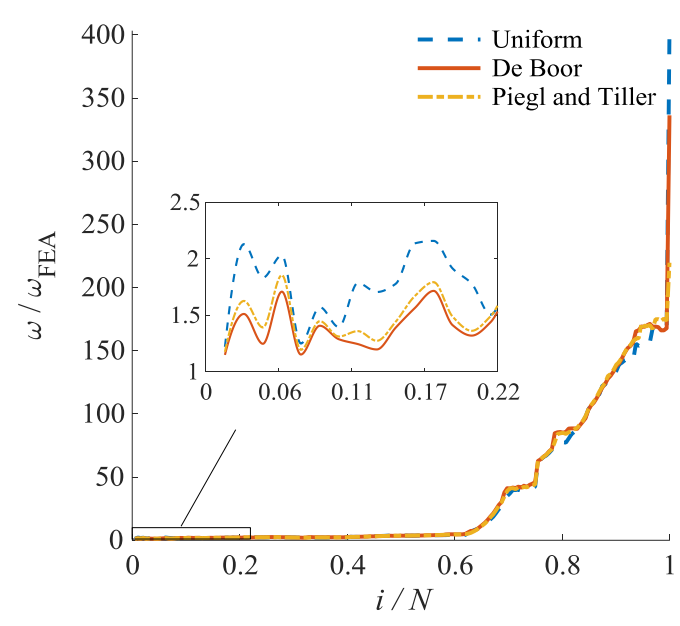

(a)

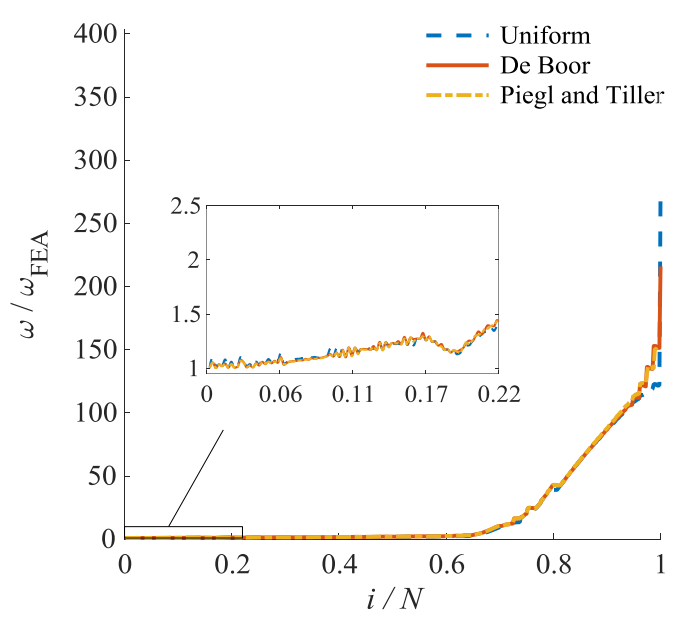

(b)

Fig. 19. Frequency spectra of the elliptic spiral beam for different knot placement techniques: (a) a coarse mesh with 50 and (b) a fine mesh with 150 control points

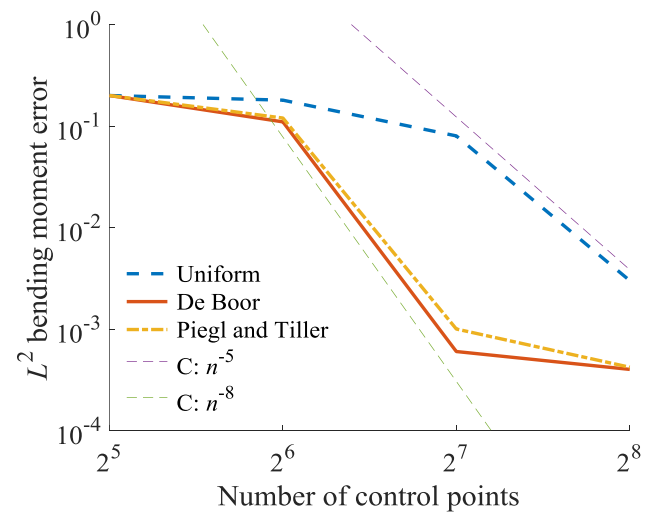

Fig. 20. Relative $L^{2}$-norm errors of bending moments for the elliptic spiral beam, obtained by cubic basis functions and different knot placement algorithms 


\subsection{The Lissajous Beam}

The Lissajous curve is a graph of complex harmonic motion in space that is described by the following analytical equations:

$$
\begin{aligned}
& x=\cos (3 t) \\
& y=\sin (2 t) \\
& z=\sin (7 t)
\end{aligned}
$$

For modeling the third case study, 1000 data points were considered for isogeometric analysis of the Lissajous curved beam (some of them are shown in Fig. 21), noting that he curve is clamped at the left end and undergoes a point load of $200 \mathrm{KN}$ in the $z$-direction at the right end. The effect of different knot placement techniques on the cubic basis functions and respective knot positions on the knot vector of the Lissajous example are depicted in Fig. 22 for a coarse mesh with 40 control points.

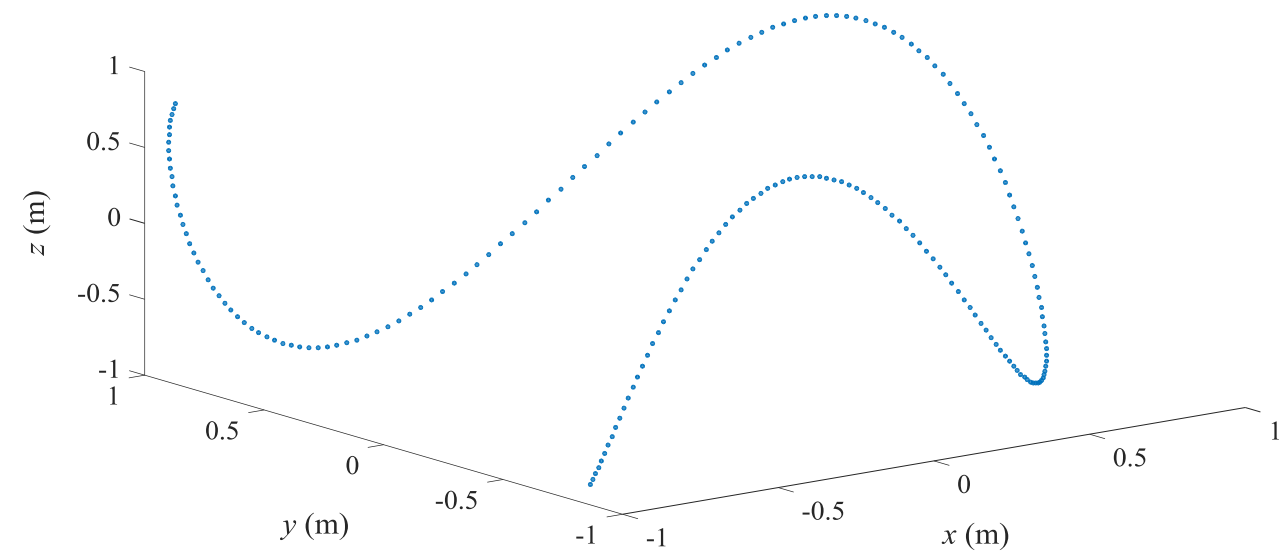

Fig. 21. Input data points of the Lissajous beam

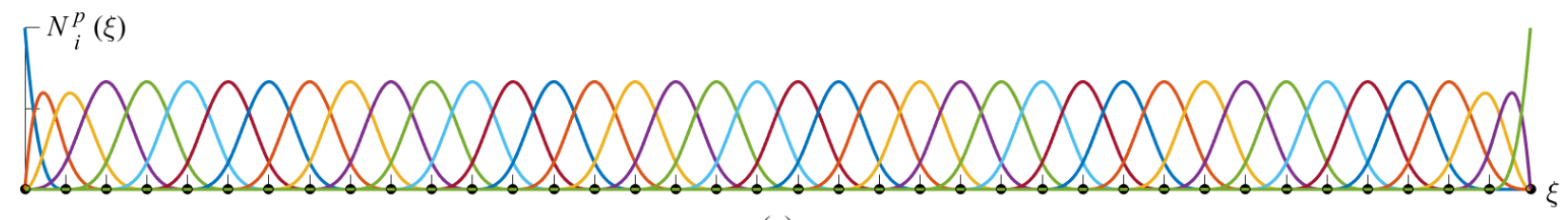

(a)

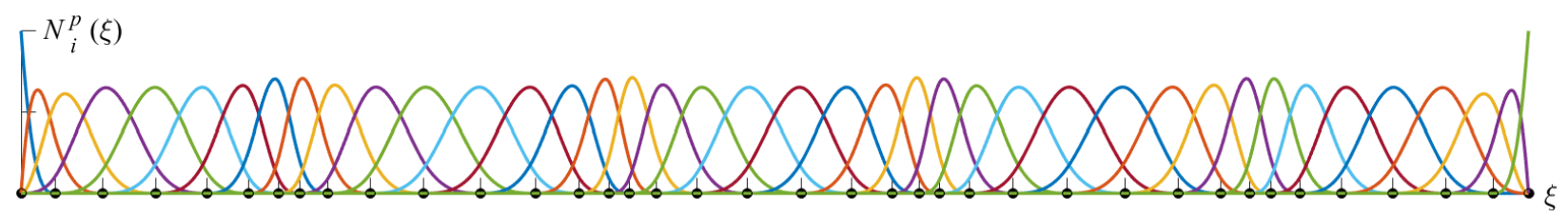

(b)

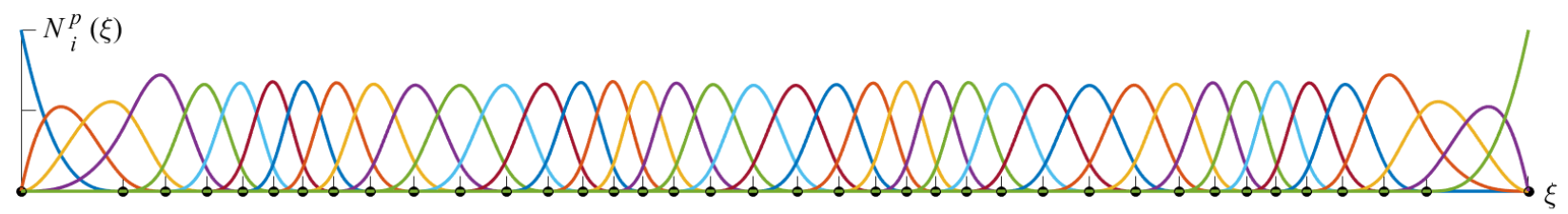

(c)

Fig. 22. Cubic basis function plots and respective knot positions for a coarse mesh (with 40 control points) of the Lissajous beam: (a) uniform knot placement, (b) De Boor's algorithm, (c) Piegl and Tiller's algorithm 
The same results as previous examples are presented in Fig. 23 and Fig. 24. The general conclusions obtained in the previous examples can be inferred again (and magnified) in the Lissajous complex curved beam, which are listed as follows:

- The De Boor's knot placement technique is, in overall, better than other two studied knot placement algorithms in isogeometric analysis-aware modeling.

- In general, the uniform knot placement method is significantly less accurate (particularly with a lower convergence rate).

- As discussed earlier, convergence to exact solutions in eigenfrequency and deflection errors of freeform case studies hardly obtainable, because no analytical solution does exist for such examples and the reference solution is also a numerical one obtained by an overkill FEA simulation.

- When it comes to curve fitting problems, we can achieve lower errors by increasing the number of control points (i.e., DOFs). In this case, the fitting error is computed as the Euclidean distance between input data points and the fitted curve. As we stated in $\$ 2.2$, zero fitting error is guaranteed by setting the number of control points equal to data points, so that the curve approximation is converted to interpolation that is not always of interest in curve fitting problems (see, e.g. [38, 44, 48]).

The plot of full frequency spectra for the third case study is also depicted in Fig. 25. The presented results show the same trend discussed in previous examples (i.e., more precisely, almost the same accuracy at higher frequencies and outliers for all knot placement techniques, and the superior behavior of the De Boor's algorithm in lower frequencies and for coarser meshes). The poor performance of the uniform knot vector in lower frequencies is particularly evident in this example.

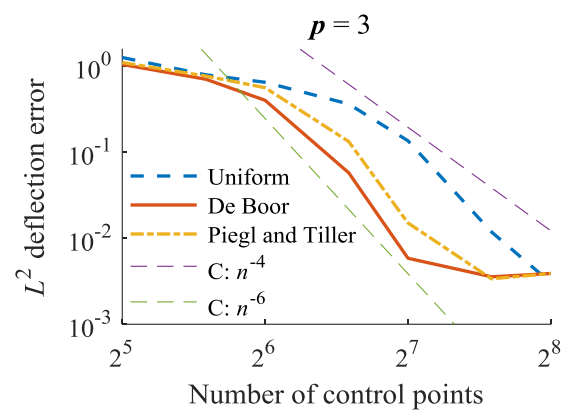

Number of control points

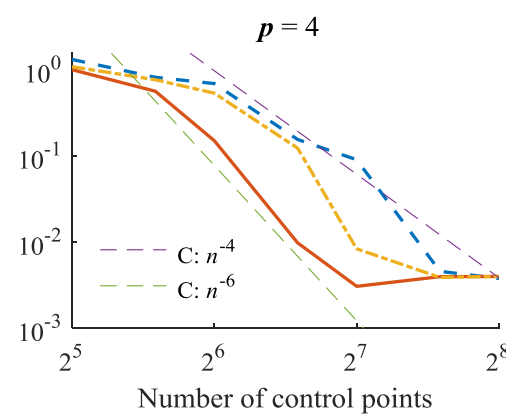

(a)

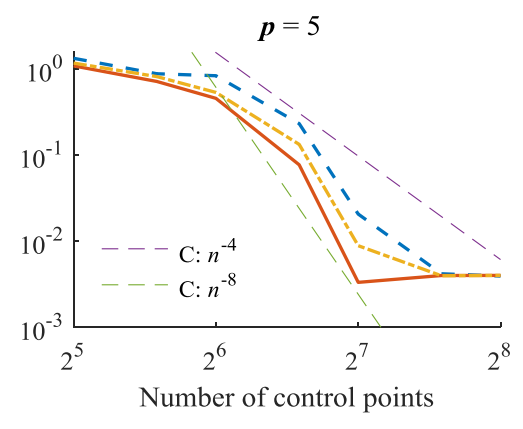

Number of control points 

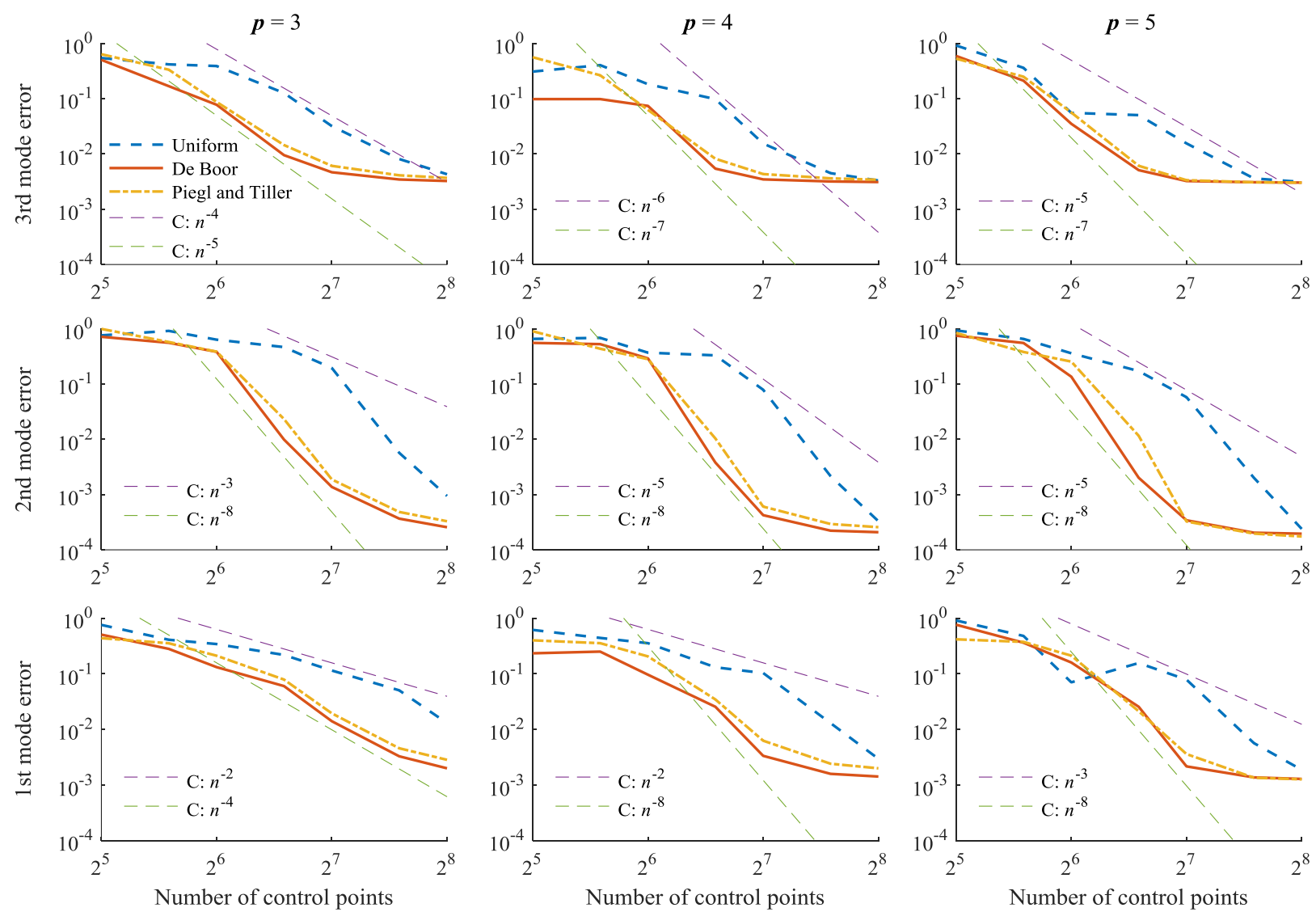

(b)

Fig. 23. Comparison of different knot placement techniques in (a) $L^{2}$-norm deflection error and (b) natural frequency analysis of the Lissajous beam: computation error vs. number of approximating control points for different spline degrees
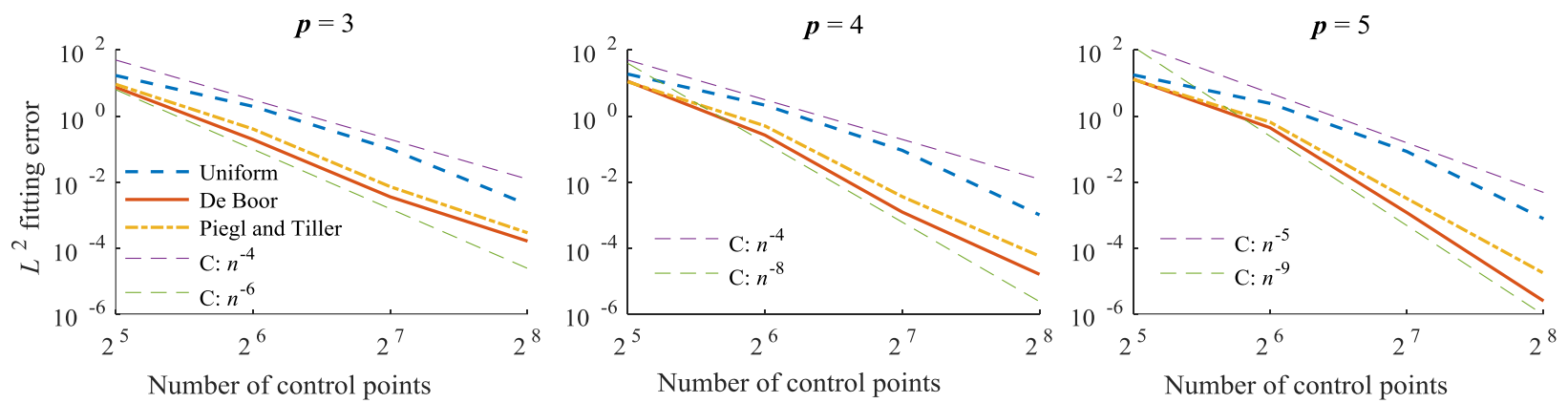

Fig. 24. $L^{2}$-norm fitting error of the Lissajous beam vs. number of approximating control points for different knot placement techniques and different spline degrees 


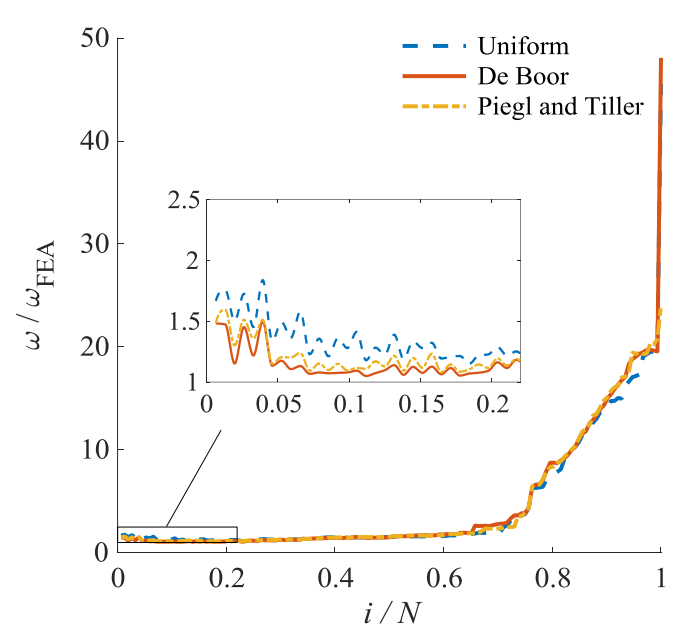

(a)

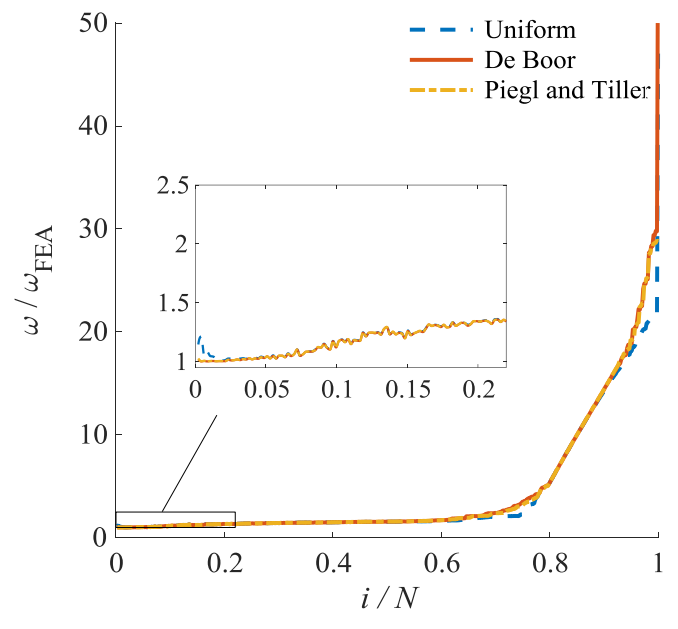

(b)

Fig. 25. Frequency spectra of the Lissajous beam for different knot placement techniques: (a) a coarse mesh with 40 and (b) a fine mesh with 140 control points

Finally, the relative $L^{2}$-norm errors of bending moments for the Lissajous beam, obtained by cubic basis functions and different knot placement algorithms, are shown in Fig. 26.

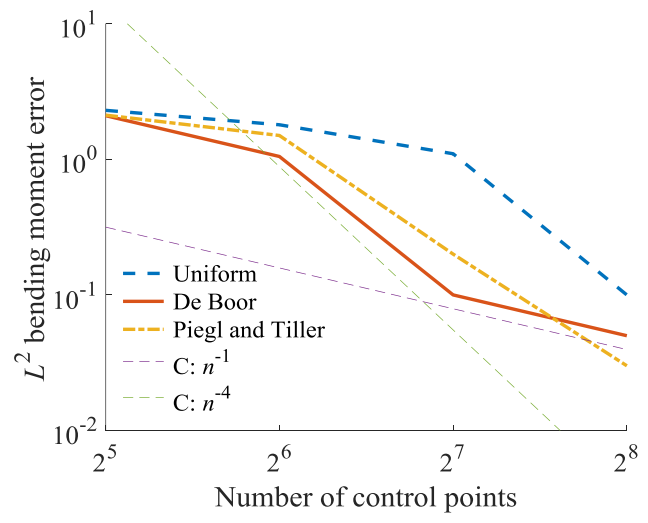

Fig. 26. Relative $L^{2}$-norm errors of bending moments for the Lissajous beam, obtained by cubic basis functions and different knot placement algorithms

\section{Supplementary Investigations}

In this section, we perform some additional investigations to demonstrate how the knot placement techniques can deal with different geometric aspects of the IGA framework. In our assessments, we first consider the situations in which different data distributions of the geometry of interest are input. Then, the presence of noise in data distribution and its impact on IGA results are studied, followed by a comparison between the geometry construction by curve fitting and typical refinements techniques. Finally, we 
investigate how the fitting error obtained by De Boor's algorithm can be close to the optimal knot placement in which the knot positions are found by an optimization procedure.

\subsection{Different Distributions of Data Points}

In order to investigate how the IGA results may depend on the initial distribution of the input data points, two case studies of this article, namely, the Tschirnhausen and Lissajous beams, are revisited here with different data distributions. These distributions are obtained by different increments of parameter $t$ in the analytical expression of the respective geometries. Although geometry construction by curve fitting seems to be sensitive to initial data distribution, the IGA results are shown to be quite insensitive to how the input data are provided when the quasi-linear parameterization of the chord-length parameter selection scheme is combined with De Boor's knot placement technique. In the first example of this section (see Fig. 27), two different data distributions of the Tschirnhausen beam, in which the input data are biased to the right and left ends of the beam (Cases 1 and 2, respectively), are compared to the original model (Case 3), studied earlier in $\$ 4.1$, while cubic basis functions are considered. It should be noted that for different natural frequencies, the convergence behavior might be different. The characteristics of the basis functions and the positions of knots (obtained through data fitting process) with respect to different mode shapes are our main justification for such a trend. In the second example, as shown in Fig. 28, we study different knot placement algorithms on three different data distributions of the Lissajous example in which the input data are biased to the ends (Case 1) and center (Case 2) of the beam and compared to the original model (Case 3). The eigenfrequency and fitting errors obtained by cubic bases for different data distributions are also reported in the figure. It can be seen that the De Boor's algorithm can generally lead to more accurate IGA results.
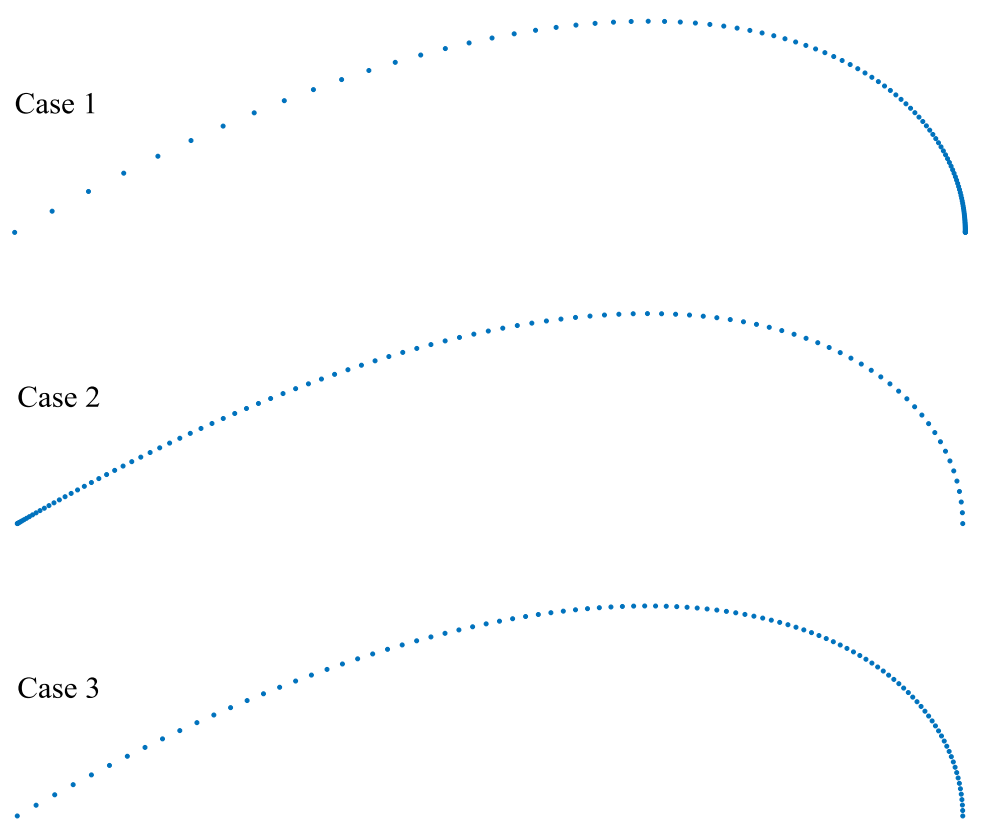

(a) 

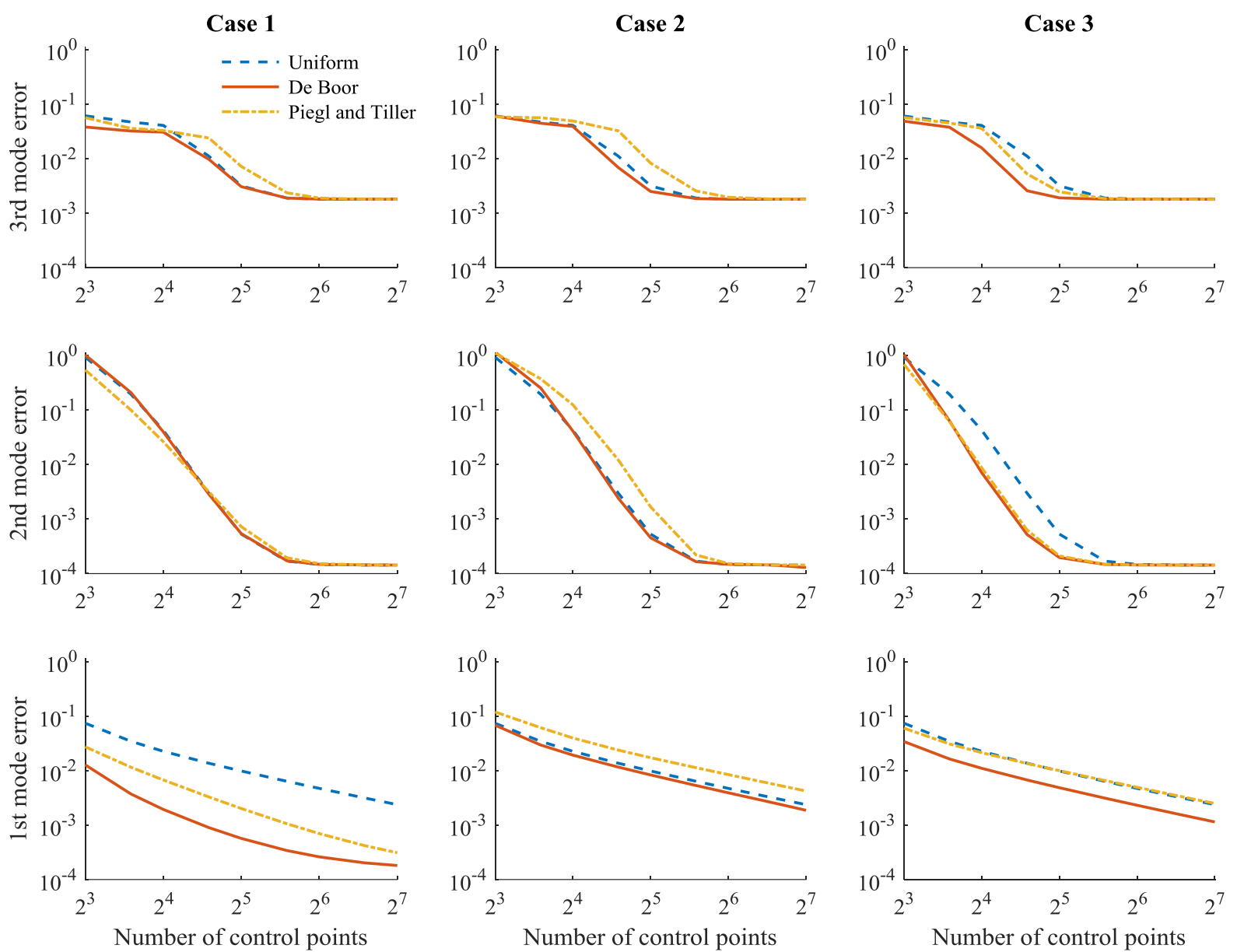

(b)

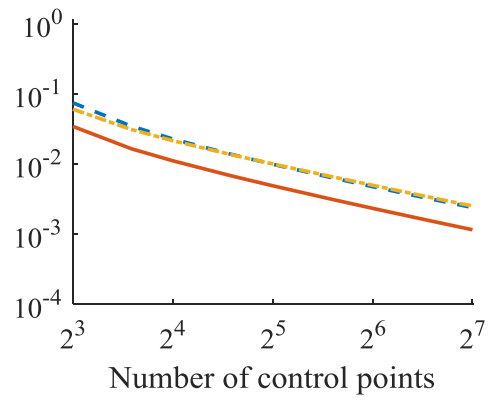

Fig. 27. (a) Different data distributions of the Tschirnhausen beam and (b) their impacts on first three eigenfrequencies obtained by cubic bases; Note: Case 3 in part (b) is the reproduction of Fig. $10 \mathrm{~b}$ for $p=3$

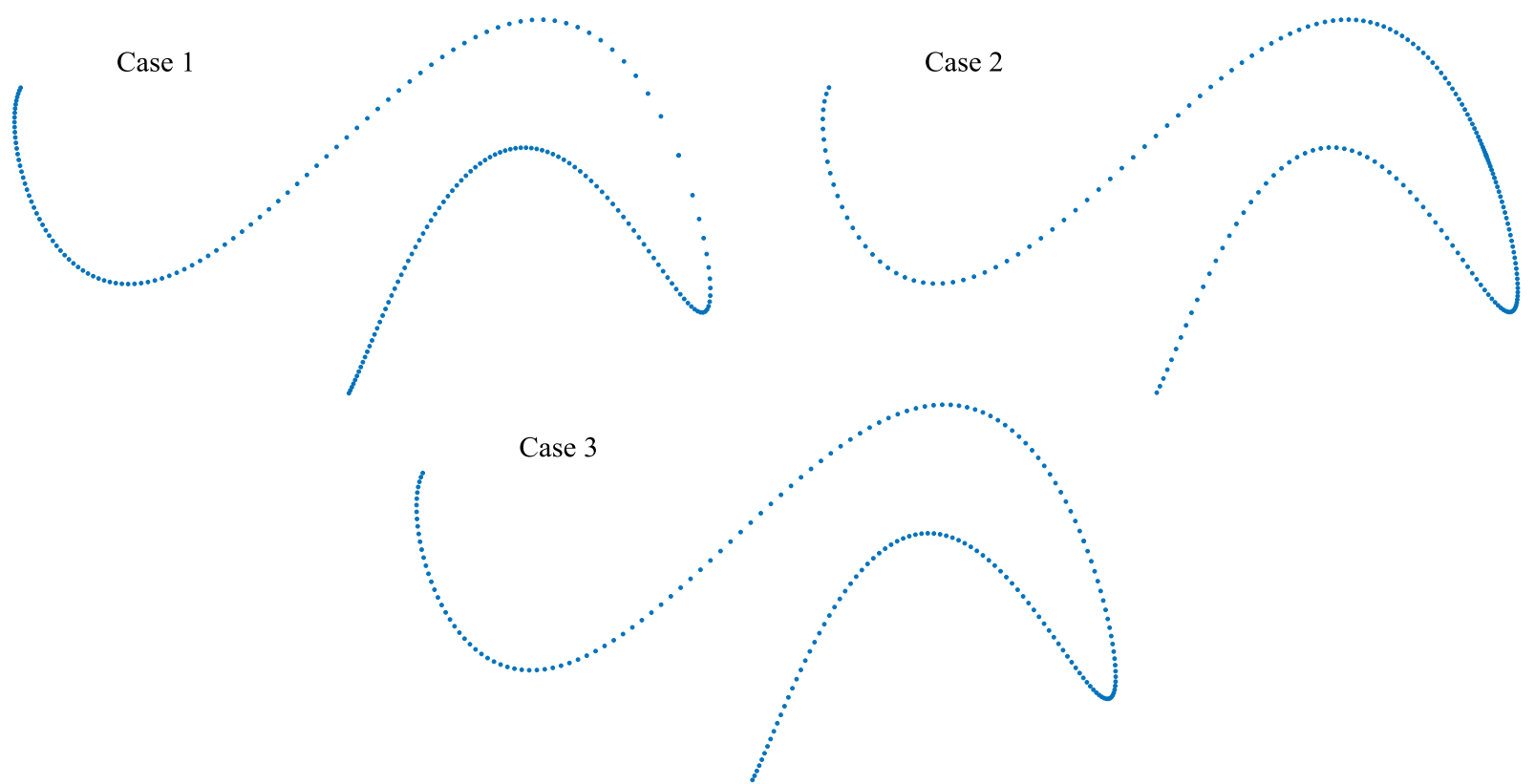



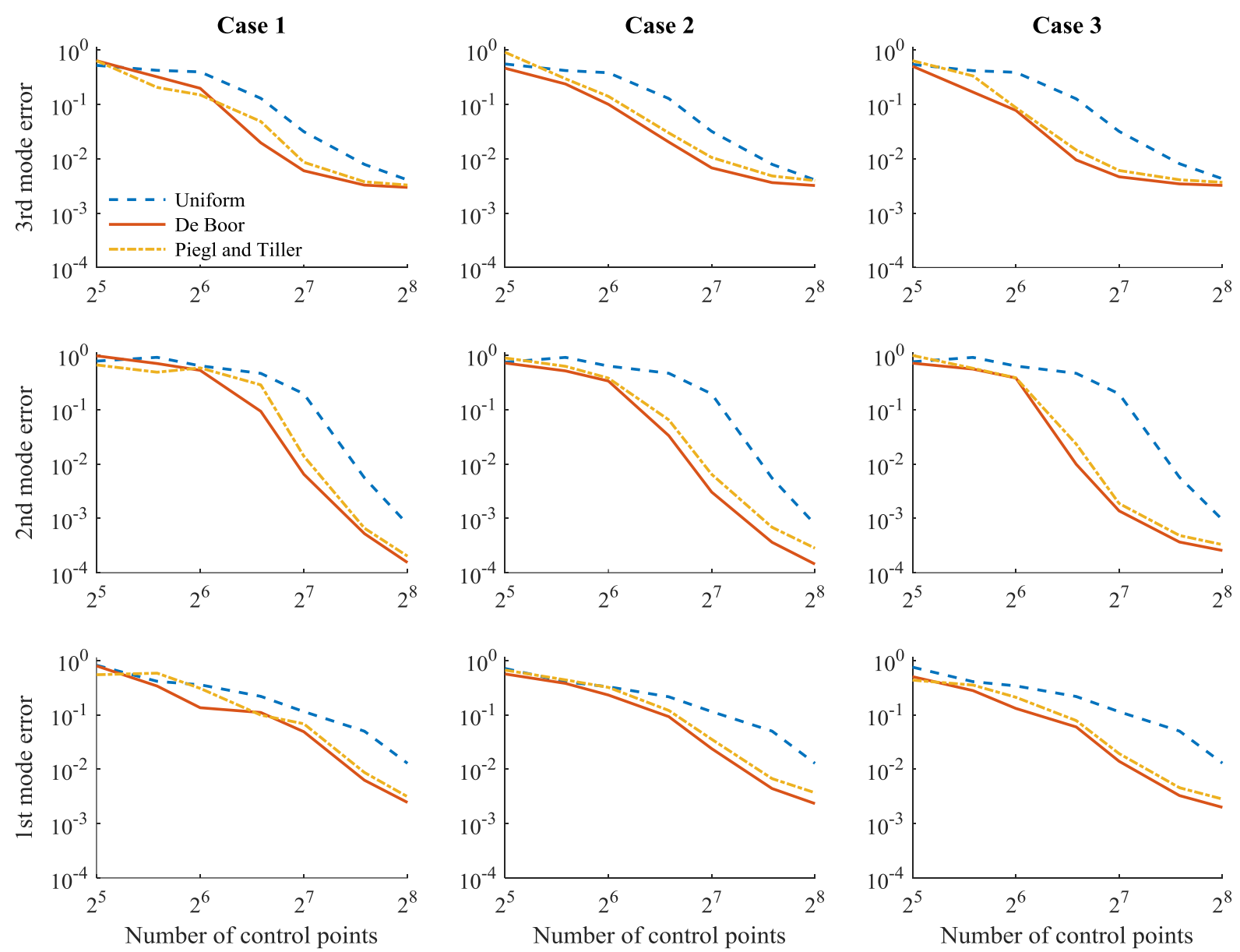

(b)
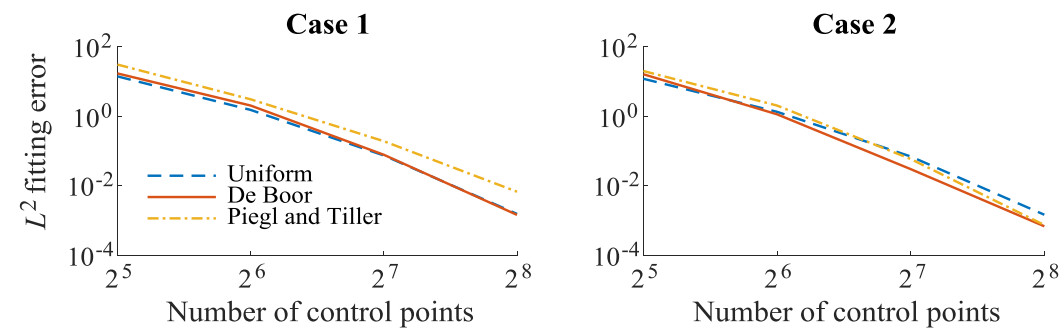

(c)

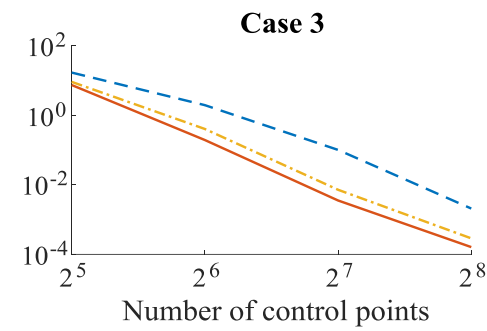

Fig. 28. (a) Different data distributions of the Lissajous beam, (b) their impacts on first three eigenfrequencies and (c) curve fitting errors with cubic bases; Note: Case 3 in parts (b) and (c) are the reproductions of Fig. $23 \mathrm{~b}$ and Fig. 24 for $p=3$

\subsection{Effect of Noisy Data}

In order to investigate how a noisy dataset may affect the IGA results, we herein study the elliptic spiral beam with noisy distribution of input data points. Fig. 29a depicts some input data with noise levels of $1 \%$ and 3\%. By studying the effect of different noise levels on first three eigenfrequencies obtained by cubic basis functions and different knot placement algorithms (Fig. 29b), it is indicated that increasing the number 
of control points up to a certain level, depending on the noise magnitude, may improve the IGA results. However, with more control points, it is clear that the fitted B-spline curve would be more flexible and follow the noisy data with lower fitting error. Consequently, the fitted curve deviates more from the original geometry and the IGA errors increase compared to the reference solution. It is also clear in the figure that De Boor's knot placement algorithm generally leads to lower simulation errors.
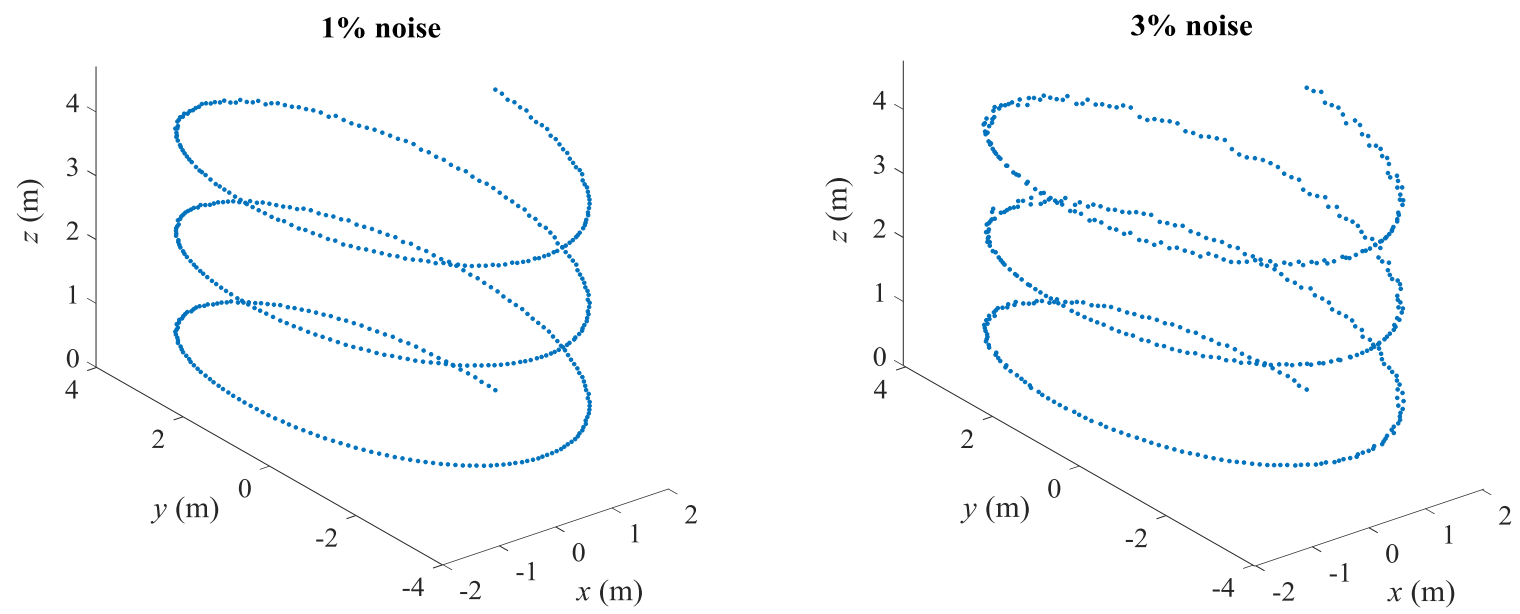

(a)
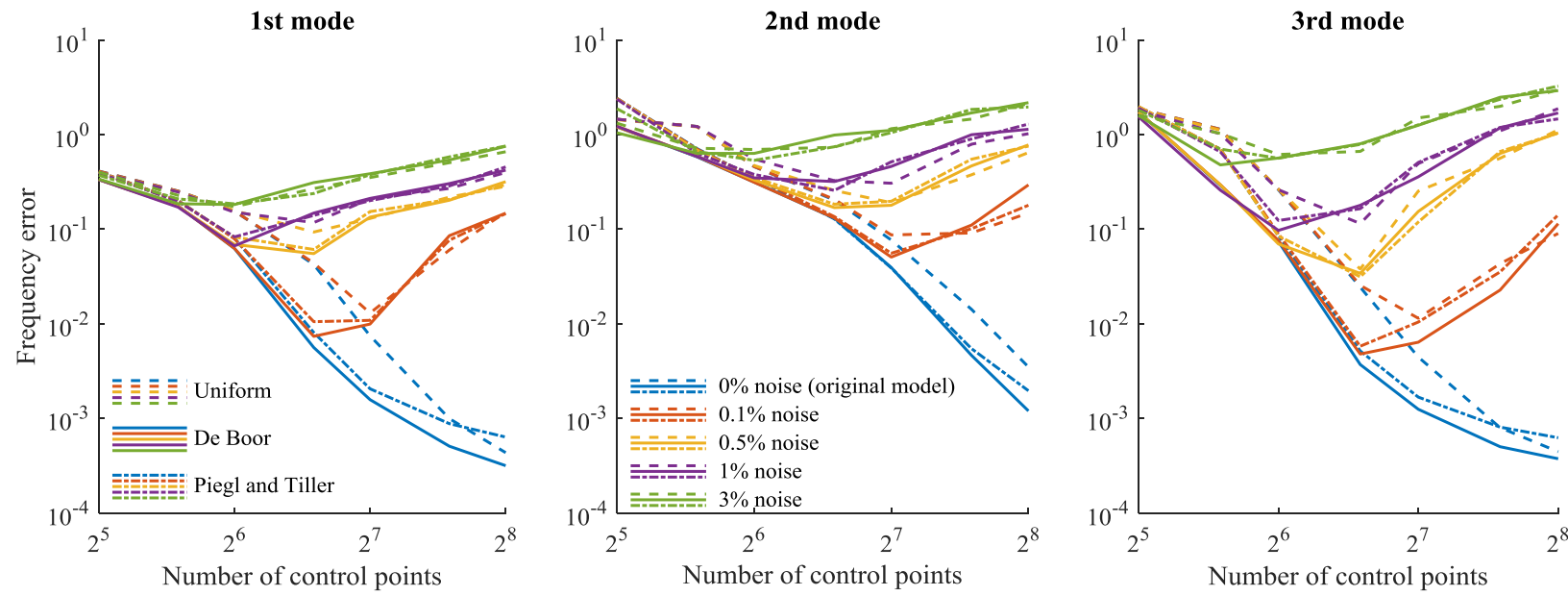

(b)

Fig. 29. (a) Input data of elliptic spiral beam with $1 \%$ and $3 \%$ noise levels, (b) effect of different noise levels on first three eigenfrequencies of elliptic spiral beam obtained by cubic basis functions and different knot placement algorithms

\subsection{Typical h-Refinement vs. Curve Fitting}

In the presented IGA framework with curved beams given in terms of input data points, the geometry is obtained by curve approximation and the mesh refinement is performed inherently in the fitting process by selecting the appropriate number of control points. Hence, both fitting and frequency (or static) analysis 
errors will generally be reduced by employing more control points. However, if the geometry is constructed by lower (i.e., not enough) number of control points and then the appropriate mesh density is to be obtained by a typical $h$-refinement, the IGA errors may converge to larger values compared to those obtained by the same number of control points in curve fitting. This is because for the former, the geometry inherits a larger fitting error and deviates more from the reference solution. This comparison is illustrated in Fig. 30 for the first eigenfrequency of the elliptic spiral beam, noting that for the $h$-refined geometries, the initial fitted curve had 32 control points. It is also clear that one can simply perform curve fitting with more control points and employ a knot placement technique that produces a better fit; and then, use typical $h$-, $p$ - and $k$ refinements on the same geometry to make the analysis results as accurate as desired.
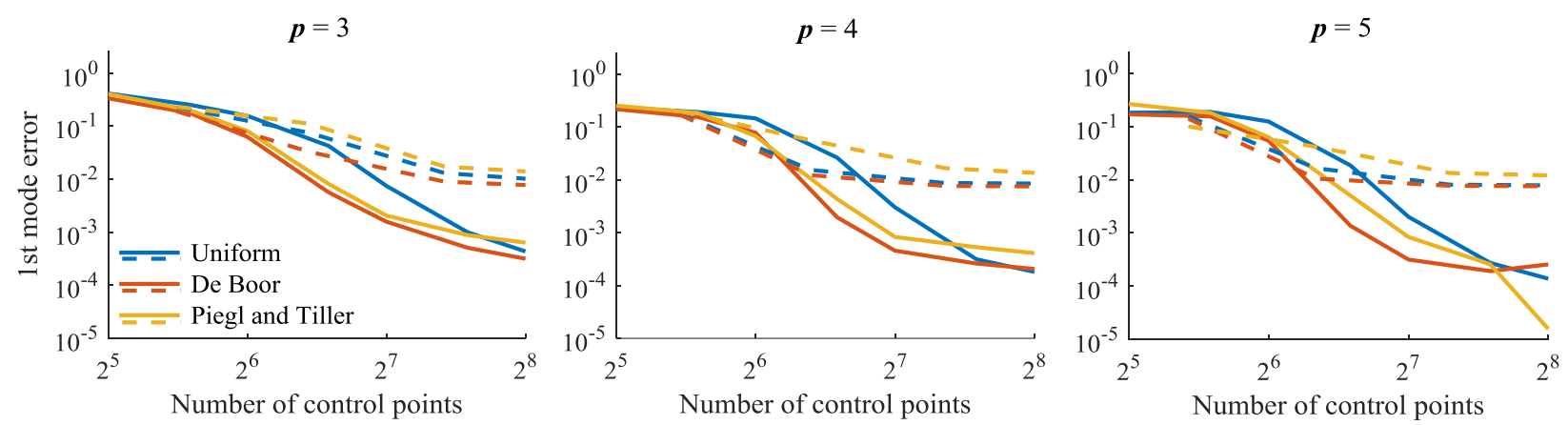

Fig. 30. Comparison of the first eigenfrequency results of the elliptic spiral beam when the geometry is obtained by curve fitting (solid lines) and by $h$-refinement (dashed lines)

\subsection{Comparing to Optimal Knot Placement}

In order to obtain the best knot placement for curve approximation, we can consider internal knots of the knot vector as unknowns that should be found along with control points in the curve fitting process. The procedure, however, entails performing an optimization to find the best knot positions. Although we may achieve a better curve fit in this way, we need to spend more time to perform the optimization (about 100 times greater than that required for other three algorithms). On the hand, as shown in Fig. 31 for the Tschirnhausen example, the fitting error obtained by De Boor's algorithm is very close to the optimal knot placement error, especially when the number of control points increases. In this figure, a gradient-based optimization with sequential quadratic programming (SQP) is employed. Consequently, it is not reasonable to employ such optimization directly in an isogeometric problem and the De Boor's method can be used instead. 


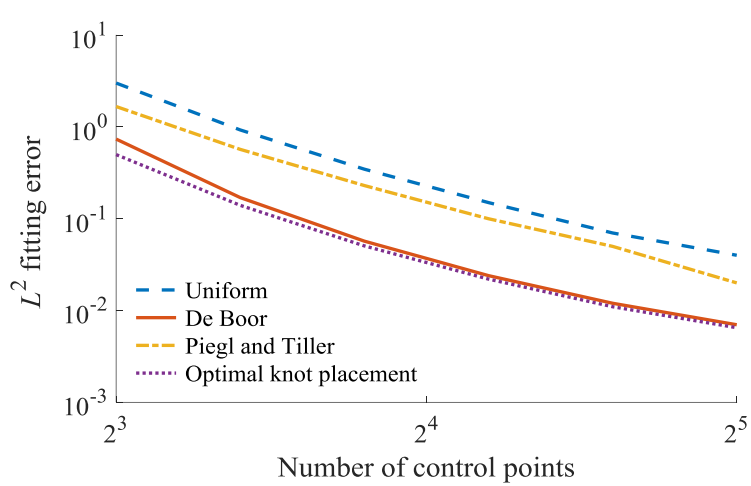

Fig. 31. Convergence of fitting error of the Tschirnhausen example obtained by the optimal and well-known knot placements

\section{Conclusions}

The effect of different knot placement techniques in geometry construction for isogeometric analysis is investigated in this study. Using the implemented IGA formulation for structural static and vibration analyses of free-form spatial curved beams, three well-known knot vector generation approaches (namely, uniformly spaced, De Boor's, and Piegl and Tiller's algorithms) are compared in the cases that the geometry needs to be constructed from input dataset by means of a curve approximation. The main message of the present research lies in the fact that employing different knot placement techniques not only affects the quality of the constructed geometry but also changes the characterization of the IGA elements and therefore the accuracy of results. Different case studies and numerical examples are presented in this paper and the results are validated against commercial finite element software. All examples revealed that the De Boor's algorithm typically leads to superior results compared to uniformly spaced and Piegl and Tiller's knot placement strategies. Interestingly, the widely used uniformly spaced knot placement technique may lead to particularly inaccurate results. Future works may include the extension of the present study to 2D and 3D problems although the authors would like to add the fact that the linear (pseudo arclength) parameterization that was adopted in this paper and works so nicely for beams is not easy to be extended to multidimensional cases.

\section{Acknowledgements}

Ali Hashemian was partially funded by the BCAM "Severo Ochoa" accreditation of excellence (SEV-20170718), and the Basque Government through the BERC 2018-2021 program.

Alessandro Reali was partially supported by the MIUR-PRIN project XFAST-SIMS (no. 20173C478N).

The authors would also like to thank the anonymous reviewers for their comments, which helped to improve the paper and led to the interesting additional studies reported in Section 5. 


\section{References}

[1] T.J.R. Hughes, J.A. Cottrell, Y. Bazilevs, Isogeometric analysis: CAD, finite elements, NURBS, exact geometry and mesh refinement, Computer Methods in Applied Mechanics and Engineering, 194 (2005) 4135-4195. http://dx.doi.org/10.1016/j.cma.2004.10.008.

[2] L. Beirão da Veiga, C. Lovadina, A. Reali, Avoiding shear locking for the Timoshenko beam problem via isogeometric collocation methods, Computer Methods in Applied Mechanics and Engineering, 241244 (2012) 38-51. http://dx.doi.org/10.1016/j.cma.2012.05.020.

[3] L. De Lorenzis, P. Wriggers, G. Zavarise, A mortar formulation for 3D large deformation contact using NURBS-based isogeometric analysis and the augmented Lagrangian method, Computational Mechanics, 49 (2012) 1-20. http://dx.doi.org/10.1007/s00466-011-0623-4.

[4] R. Bouclier, T. Elguedj, A. Combescure, On the development of NURBS-based isogeometric solid shell elements: 2D problems and preliminary extension to 3D, Computational Mechanics, 52 (2013) 1085-1112. http://dx.doi.org/10.1007/s00466-013-0865-4.

[5] G. Zhang, K. Khandelwal, Modeling of nonlocal damage-plasticity in beams using isogeometric analysis, Computers \& Structures, 165 (2016) 76-95. http://dx.doi.org/10.1016/j.compstruc.2015.12.006.

[6] S.-H. Ahn, M.-J. Choi, S. Cho, Isogeometric shape design optimization of nanoscale structures using continuum-based shell theory considering surface effects, International Journal of Mechanical Sciences, 141 (2018) 9-20. http://doi.org/10.1016/j.ijmecsci.2018.03.043.

[7] Y. Bazilevs, V.M. Calo, T.J.R. Hughes, Y. Zhang, Isogeometric fluid-structure interaction: theory, algorithms, and computations, Computational Mechanics, $43 \quad$ (2008) 3-37. http://dx.doi.org/10.1007/s00466-008-0315-x.

[8] Y. Ghaffari Motlagh, H.T. Ahn, T.J.R. Hughes, V.M. Calo, Simulation of laminar and turbulent concentric pipe flows with the isogeometric variational multiscale method, Computers \& Fluids, 71 (2013) 146-155. http://dx.doi.org/10.1016/j.compfluid.2012.09.006.

[9] J. Bueno, H. Gomez, Liquid-vapor transformations with surfactants. Phase-field model and Isogeometric Analysis, Journal of Computational Physics, 321 (2016) 797-818. http://doi.org/10.1016/j.jcp.2016.06.008.

[10] A. Hashemian, E. Lakzian, A. Ebrahimi-Fizik, On the application of isogeometric finite volume method in numerical analysis of wet-steam flow through turbine cascades, Computers \& Mathematics with Applications, (2019). http://doi.org/10.1016/j.camwa.2019.09.025.

[11] M. Yoon, S.-H. Ha, S. Cho, Isogeometric shape design optimization of heat conduction problems, International Journal of Heat and Mass Transfer, 62 (2013) 272-285. http://dx.doi.org/10.1016/j.ijheatmasstransfer.2013.02.077.

[12] Z.-P. Wang, S. Turteltaub, M. Abdalla, Shape optimization and optimal control for transient heat conduction problems using an isogeometric approach, Computers \& Structures, 185 (2017) 59-74. http://dx.doi.org/10.1016/j.compstruc.2017.02.004. 
[13] T.J.R. Hughes, J.A. Evans, A. Reali, Finite element and NURBS approximations of eigenvalue, boundary-value, and initial-value problems, Computer Methods in Applied Mechanics and Engineering, 272 (2014) 290-320. http://dx.doi.org/10.1016/j.cma.2013.11.012.

[14] D. Wang, Q. Liang, H. Zhang, A superconvergent isogeometric formulation for eigenvalue computation of three dimensional wave equation, Computational Mechanics, 57 (2016) 1037-1060. http://dx.doi.org/10.1007/s00466-016-1276-0.

[15] R. Bouclier, T. Elguedj, A. Combescure, Locking free isogeometric formulations of curved thick beams, Computer Methods in Applied Mechanics and Engineering, 245-246 (2012) 144-162. http://dx.doi.org/10.1016/j.cma.2012.06.008.

[16] O. Weeger, U. Wever, B. Simeon, Isogeometric analysis of nonlinear Euler-Bernoulli beam vibrations, Nonlinear Dynamics, 72 (2013) 813-835. http://dx.doi.org/10.1007/s11071-013-0755-5.

[17] A.-T. Luu, N.-I. Kim, J. Lee, Isogeometric vibration analysis of free-form Timoshenko curved beams, Meccanica, 50 (2015) 169-187. http://dx.doi.org/10.1007/s11012-014-0062-3.

[18] G. Balduzzi, S. Morganti, F. Auricchio, A. Reali, Non-prismatic Timoshenko-like beam model: Numerical solution via isogeometric collocation, Computers \& Mathematics with Applications, 74 (2017) 1531-1541. http://doi.org/10.1016/j.camwa.2017.04.025.

[19] S.F. Hosseini, A. Hashemian, B. Moetakef-Imani, S. Hadidimoud, Isogeometric Analysis of Free-form Timoshenko Curved Beams Including the Nonlinear Effects of Large Deformations, Acta Mechanica Sinica, 34 (2018) 728-743. http://dx.doi.org/10.1007/s10409-018-0753-4.

[20] A. Borković, S. Kovačević, G. Radenković, S. Milovanović, M. Guzijan-Dilber, Rotation-free isogeometric analysis of an arbitrarily curved plane Bernoulli-Euler beam, Computer Methods in Applied Mechanics and Engineering, 334 (2018) 238-267. http://doi.org/10.1016/j.cma.2018.02.002.

[21] A. Hashemian, S.F. Hosseini, Nonlinear bifurcation analysis of statically loaded free-form curved beams using isogeometric framework and pseudo-arclength continuation, International Journal of NonLinear Mechanics, 113 (2019) 1-16. http://dx.doi.org/10.1016/j.ijnonlinmec.2019.03.002.

[22] L. Greco, M. Cuomo, B-Spline interpolation of Kirchhoff-Love space rods, Computer Methods in Applied Mechanics and Engineering, 256 (2013) 251-269. http://dx.doi.org/10.1016/j.cma.2012.11.017.

[23] A.M. Bauer, M. Breitenberger, B. Philipp, R. Wüchner, K.U. Bletzinger, Nonlinear isogeometric spatial Bernoulli beam, Computer Methods in Applied Mechanics and Engineering, 303 (2016) 101-127. http://dx.doi.org/10.1016/j.cma.2015.12.027.

[24] G. Zhang, R. Alberdi, K. Khandelwal, On the locking free isogeometric formulations for 3-D curved Timoshenko beams, Finite Elements in Analysis and Design, 143 (2018) 46-65. http://doi.org/10.1016/j.finel.2018.01.007.

[25] G. Radenković, A. Borković, Linear static isogeometric analysis of an arbitrarily curved spatial Bernoulli-Euler beam, Computer Methods in Applied Mechanics and Engineering, 341 (2018) 360-396. http://doi.org/10.1016/j.cma.2018.07.010. 
[26] O. Weeger, B. Narayanan, M.L. Dunn, Isogeometric shape optimization of nonlinear, curved 3D beams and beam structures, Computer Methods in Applied Mechanics and Engineering, 345 (2019) 26-51. http://doi.org/10.1016/j.cma.2018.10.038.

[27] E. Marino, J. Kiendl, L. De Lorenzis, Explicit isogeometric collocation for the dynamics of threedimensional beams undergoing finite motions, Computer Methods in Applied Mechanics and Engineering, 343 (2019) 530-549. http://doi.org/10.1016/j.cma.2018.09.005.

[28] E. Marino, Isogeometric collocation for three-dimensional geometrically exact shear-deformable beams, Computer Methods in Applied Mechanics and Engineering, 307 (2016) 383-410. http://doi.org/10.1016/j.cma.2016.04.016.

[29] O. Weeger, S.-K. Yeung, M.L. Dunn, Isogeometric collocation methods for Cosserat rods and rod structures, Computer Methods in Applied Mechanics and Engineering, 316 (2017) 100-122. http://doi.org/10.1016/j.cma.2016.05.009.

[30] E. Cohen, T. Martin, R.M. Kirby, T. Lyche, R.F. Riesenfeld, Analysis-aware modeling: Understanding quality considerations in modeling for isogeometric analysis, Computer Methods in Applied Mechanics and Engineering, 199 (2010) 334-356. http://dx.doi.org/10.1016/j.cma.2009.09.010.

[31] G. Xu, B. Mourrain, R. Duvigneau, A. Galligo, Parameterization of computational domain in isogeometric analysis: Methods and comparison, Computer Methods in Applied Mechanics and Engineering, 200 (2011) 2021-2031. http://dx.doi.org/10.1016/j.cma.2011.03.005.

[32] G. Xu, B. Mourrain, R. Duvigneau, A. Galligo, Optimal analysis-aware parameterization of computational domain in 3D isogeometric analysis, Computer-Aided Design, 45 (2013) 812-821. http://dx.doi.org/10.1016/j.cad.2011.05.007.

[33] G. Xu, M. Li, B. Mourrain, T. Rabczuk, J. Xu, S.P. Bordas, Constructing IGA-suitable planar parameterization from complex CAD boundary by domain partition and global/local optimization, Computer Methods in Applied Mechanics and Engineering, 328 (2018) 175-200. http://doi.org/10.1016/j.cma.2017.08.052.

[34] H. Casquero, L. Liu, Y. Zhang, A. Reali, H. Gomez, Isogeometric collocation using analysis-suitable T-splines of arbitrary degree, Computer Methods in Applied Mechanics and Engineering, 301 (2016) 164186. http://doi.org/10.1016/j.cma.2015.12.014.

[35] M. Aigner, C. Heinrich, B. Jüttler, E. Pilgerstorfer, B. Simeon, A.-V. Vuong, Swept Volume Parameterization for Isogeometric Analysis, Mathematics of Surfaces XIII: 13th IMA International Conference York, UK, Springer, Berlin, Germany, 2009, pp. 19-44.

[36] S. Morganti, F. Auricchio, D.J. Benson, F.I. Gambarin, S. Hartmann, T.J.R. Hughes, A. Reali, Patientspecific isogeometric structural analysis of aortic valve closure, Computer Methods in Applied Mechanics and Engineering, 284 (2015) 508-520. http://doi.org/10.1016/j.cma.2014.10.010.

[37] S.F. Hosseini, A. Hashemian, A. Reali, On the application of curve reparameterization in isogeometric vibration analysis of free-from curved beams, Computers \& Structures, 209 (2018) 117-129. http://doi.org/10.1016/j.compstruc.2018.08.009.

[38] L. Piegl, W. Tiller, The NURBS Book, 2nd ed., Springer-Verlag, New York, NY, 1997. 
[39] S.F. Hosseini, B. Moetakef-Imani, S. Hadidi-Moud, B. Hassani, The effect of parameterization on isogeometric analysis of free-form curved beams, Acta Mechanica, 227 (2016) 1983-1998. http://dx.doi.org/10.1007/s00707-016-1610-9.

[40] J.A. Cottrell, A. Reali, Y. Bazilevs, T.J.R. Hughes, Isogeometric analysis of structural vibrations, Computer Methods in Applied Mechanics and Engineering, 195 (2006) 5257-5296. http://dx.doi.org/10.1016/j.cma.2005.09.027.

[41] S.F. Hosseini, B. Moetakef-Imani, S. Hadidi-moud, B. Hassani, Pre-bent Shape Design of Full FreeForm Curved Beams Using Isogeometric Method and Semi-Analytical Sensitivity Analysis, Structural and Multidisciplinary Optimization, 58 (2018) 2621-2633. http://doi.org/10.1007/s00158-018-2041-0.

[42] A. Hashemian, S.F. Hosseini, An integrated fitting and fairing approach for object reconstruction using smooth NURBS curves and surfaces, Computers \& Mathematics with Applications, 76 (2018) 1555-1575. http://doi.org/10.1016/j.camwa.2018.07.007.

[43] B.M. Imani, S.A. Hashemian, NURBS-Based Profile Reconstruction using Constrained Fitting Techniques, Journal of Mechanics, 28 (2012) 407-412. http://dx.doi.org/10.1017/jmech.2012.71.

[44] A. Hashemian, S.F. Hosseini, S.N. Nabavi, Kinematically Smoothing Trajectories by NURBS Reparameterization - An Innovative Approach, Advanced Robotics, 31 (2017) 1296-1312. http://dx.doi.org/10.1080/01691864.2017.1396923.

[45] H. Park, B-spline surface fitting based on adaptive knot placement using dominant columns, ComputerAided Design, 43 (2011) 258-264. http://doi.org/10.1016/j.cad.2010.12.001.

[46] F. Pérez-Arribas, R. Pérez-Fernández, A B-spline design model for propeller blades, Advances in Engineering Software, 118 (2018) 35-44. http://doi.org/10.1016/j.advengsoft.2018.01.005.

[47] L.A. Piegl, W. Tiller, Surface approximation to scanned data, The Visual Computer, 16 (2000) 386395. http://doi.org/10.1007/p100013393.

[48] G. Farin, Curves and Surfaces for CAGD, 5th ed., Morgan Kaufmann, San Francisco, CA, 2002.

[49] R. Echter, M. Bischoff, Numerical efficiency, locking and unlocking of NURBS finite elements, Computer Methods in Applied Mechanics and Engineering, 199 (2010) 374-382. http://doi.org/10.1016/j.cma.2009.02.035.

[50] S.J. Lee, K.S. Park, Vibrations of Timoshenko beams with isogeometric approach, Applied Mathematical Modelling, 37 (2013) 9174-9190. http://dx.doi.org/10.1016/j.apm.2013.04.034.

[51] A.P. Nagy, M.M. Abdalla, Z. Gürdal, Isogeometric design of elastic arches for maximum fundamental frequency, Structural and Multidisciplinary Optimization, $43 \quad$ (2011) 135-149. http://dx.doi.org/10.1007/s00158-010-0549-z. 